\title{
THE SZEGÖ KERNEL AS A SINGULAR INTEGRAL KERNEL ON A FAMILY OF WEAKLY PSEUDOCONVEX DOMAINS
}

\author{
KATHARINE PERKINS DIAZ
}

\begin{abstract}
The Szegö kernels on the weakly pseudoconvex domains $\left\{\operatorname{Im} z_{2}>\left|z_{1}\right|^{2 k}\right\}$, $k \in Z^{+}$, have been computed by Greiner and Stein. After constructing a global, nonisotropic pseudometric suitable for Calderón-Zygmund singular integral theory on the boundaries of the domains, we study principal value operators associated to these Szegö kernels. We prove that the principal value operators are bounded on $L^{p}$, for $1<p<\infty$, and that they preserve certain nonisotropic Lipschitz classes. We then derive a Plemelj formula that relates the principal value operators to the Szegö projections. From this formula we deduce that the Szegö projections are also bounded on $L^{p}$, for $1<p<\infty$, and that they preserve the same nonisotropic Lipschitz classes.
\end{abstract}

1. Introduction. We show that the Szegö projection on the weakly pseudoconvex domains $H_{k}=\left\{\left(z_{1}, z_{2}\right) \in \mathbf{C}^{2}: \operatorname{Im} z_{2}>\left|z_{1}\right|^{2 k}\right\}, k$ a positive integer, is bounded on $L^{p}\left(\partial H_{k}\right)$, for $1<p<\infty$, and that it preserves certain nonisotropic Lipschitz classes $\Gamma_{\alpha}$, for $0<\alpha<1$.

In a paper on singular integrals on homogeneous spaces [KoV], Korányi and Vági showed that on the ball and on the generalized upper half-space in $\mathbf{C}^{n}$, the Szegö projection is bounded on $L^{p}$ of the boundary and that it preserves $\Gamma_{\alpha}$, for $0<\alpha<1$. Phong and Stein [PS] showed boundedness on $L^{p}, 1<p<\infty$, and preservation of $\Gamma_{\alpha}$ for $0<\alpha<\infty$ for the Szegö projection on all bounded, strictly pseudoconvex domains. Phong and Stein [PS] and, independently, Ahern and Schneider [AhS] showed that the standard, that is isotropic, Lipschitz classes $\Lambda_{\alpha}$, $0<\alpha<\infty$, are also preserved by the Szegö projection on bounded, strictly pseudoconvex domains. Bonami and Lohoué $[\mathbf{B L}]$ considered the weakly pseudoconvex domains $D_{\beta}=\left\{z \in \mathbf{C}^{n}:\left|z_{1}\right|^{2 / \beta_{1}}+\cdots+\left|z_{n}\right|^{2 / \beta_{n}}<1\right\}, \quad \beta_{1}, \ldots, \beta_{n} \in(0,1]$. They showed that the Szegö projections on $D_{\beta}$ are bounded on $L^{p}\left(\partial D_{\beta}\right), 1<p<\infty$.

Our work here is similar to parts of [KoV, FS, and NS]. That is, we treat the Szegö kernel from the point of view of singular integral theory on $\partial H_{k}$. The Szegö kernel of a domain in $\mathbf{C}^{n}$ possesses a nonisotropy that reflects the splitting of the tangent space of the domain into a complex tangent space and a remaining direction. Therefore, a nonisotropic singular integral theory that also reflects this splitting of the tangent space is used. In the classical case [S1], the main estimate on a singular

Received by the editors April 29, 1986.

1980 Mathematics Subject Classification. Primary 42B20, 32A25.

1987 American Mathematical Society $0002-9947 / 87 \$ 1.00+\$ .25$ per page 
integral kernel is the Calderón-Zygmund estimate

$$
\left|\nabla_{x} K(x, y)\right| \leqslant \frac{1}{|x-y| \operatorname{Vol} B_{x}(|x-y|)} .
$$

The necessary nonisotropic singular integral theory for the Szegö kernel is obtained by replacing Euclidean distance with a nonisotropic pseudometric. On the boundaries of bounded, strongly pseudoconvex domains the suitable pseudometric is constructed by taking ordinary Euclidean distance in complex tangent directions and the square root of Euclidean distance in the remaining tangent direction. See [KoV, FS, NS, and A1]. On the boundaries of weakly pseudoconvex domains matters are complicated by the fact that the nature of the nonisotropy of the Szegö kernel $S(x, \cdot)$ depends on the pseudoconvexity type of the point $x$ and also on the type of nearby points. Hence, on domains that are not strongly pseudoconvex (i.e. type two everywhere) the necessary pseudometric is not only nonisotropic, but its nonisotropy varies from point to point. Nagel, Stein, and Wainger in [NSW1 and NSW2] have defined and analyzed such a pseudometric in a local setting. However, we require global control on our kernel. Hence, we have used the Szegö kernel itself as a guide to construct a global pseudometric on $\partial H_{k}$. Much of our work consists of (1) showing that our formula does give a pseudometric with respect to which singular integral theory can be carried out and (2) estimating the Szegö kernel in terms of that pseudometric. Once these have been accomplished the $L^{p}$ and Lipschitz regularity follow in a fairly straightforward way from standard arguments.

$\S 2$ contains definitions and notation. $\$ 3$ contains the definition and basic properties of the pseudometric that we use. It is the properties derived in $\$ 3$ that allow us to adapt classical singular integral theory to the context of our domain, $\partial H_{k}$, and our pseudometric. The facts obtained in $\S 3$ also show that the pseudometric is crudely the right size for studying the Szegö kernel. $\$ 4$ contains a characterization of the pseudometric in terms of curves whose tangents always lie in the complex tangent space. It is from this characterization that we obtain a Calderon-Zygmund condition on the Szegö kernel. $\S 5$ contains theorems about singular integral operators. $\S 6$ contains the estimates on the kernel and our main results, namely Theorems 6.4.1, 6.4.2, 6.5.4, and 6.5.5.

2. The domains $H_{k}$ and their Szegö kernels. The domains we study are $H_{k}=$ $\left\{\left(z_{1}, z_{2}\right) \in \mathbf{C}^{2}: \operatorname{Im} z_{2}>\left|z_{1}\right|^{2 k}\right\}, k$ a positive integer. The boundary of $H_{1}, \partial H_{1}$, is strongly pseudoconvex everywhere. For $k>1, \partial H_{k}$ is strongly pseudoconvex for $z_{1} \neq 0$ and weakly pseudoconvex of type $2 k$ in the sense of Kohn [Ko] for $z_{1}=0$. Thus the set $\left\{z_{1}=0=\operatorname{Im} z_{2}, \operatorname{Re} z_{2} \in \mathbf{R}\right\}$ forms a real line of points in $\partial H_{k}$ of type $2 k$.

Note that $\partial H_{k}=\left\{\left(z_{1}, z_{2}\right) \in \mathbf{C}^{2}: \operatorname{Im} z_{2}=\left|z_{1}\right|^{2 k}\right\}$ is naturally parametrized by $z_{1}$ and $\operatorname{Re} z_{2}$. We use the following notation.

(2.1.1 a) Points in $\partial H_{k}$ are denoted $\zeta, \omega, \nu$ etc.

$$
\begin{array}{lll}
\zeta=\left(z_{1}, z_{2}\right) \sim(z, t), & z=z_{1} \in \mathbf{C}, & t=\operatorname{Re} z_{2} \in \mathbf{R} \\
\omega=\left(w_{1}, w_{2}\right) \sim(w, s), & w=w_{1} \in \mathbf{C}, & s=\operatorname{Re} w_{2} \in \mathbf{R} \\
\nu=\left(u_{1}, u_{2}\right) \sim(u, r), & u=u_{1} \in \mathbf{C}, & r=\operatorname{Re} u_{2} \in \mathbf{R} .
\end{array}
$$


Sometimes we write

$$
\zeta=(z, t)=(x+i y, t), \quad z=x+i y .
$$

Occasionally we refer to points inside $H_{k}$. We continue to use $\zeta, \omega$. Then, $(2.1 .1 \mathrm{c})$

$$
\begin{aligned}
& \zeta=\left(z_{1}, z_{2}\right)=\left(z, t+i \operatorname{Im} z_{2}\right)=\left(z, t+i\left(|z|^{2 k}+\mu\right)\right) ; \quad \operatorname{Im} z_{2}=\left|z_{1}\right|^{2 k}+\mu, \\
& \omega=\left(w_{1}, w_{2}\right)=\left(w, s+i \operatorname{Im} w_{2}\right)=\left(w, s+i\left(|w|^{2 k}+\eta\right)\right) ; \quad \operatorname{Im} w_{2}=\left|w_{1}\right|^{2 k}+\eta .
\end{aligned}
$$

The complex tangent space to $\partial H_{k}$, which we denote $T^{c}$, is spanned by the real and imaginary parts of the holomorphic tangent vector

$$
Z=\frac{\partial}{\partial z_{1}}+i 2 k z_{1}^{k-1} \bar{z}_{1}^{k} \frac{\partial}{\partial z_{2}}, \text { where } \frac{\partial}{\partial z}=\frac{1}{2}\left(\frac{\partial}{\partial x}-i \frac{\partial}{\partial y}\right) .
$$

Using the notation (2.1.1) and restricting to $\partial H_{k}$,

$$
Z=\frac{\partial}{\partial z}+i k z^{k-1} \bar{z}^{k} \frac{\partial}{\partial t},
$$

$$
\begin{aligned}
& \operatorname{Re} Z=\frac{1}{2} \frac{\partial}{\partial x}+k\left(x^{2}+y^{2}\right)^{k-1} y \frac{\partial}{\partial t}, \quad \text { and } \\
& \operatorname{Im} Z=-\frac{1}{2} \frac{\partial}{\partial y}+k\left(x^{2}+y^{2}\right)^{k-1} x \frac{\partial}{\partial t} .
\end{aligned}
$$

At the weakly pseudoconvex points, where $z=0, T^{c}$ is simply the $z$ plane. For other $z, T^{c}$ is tilted slightly from the $z$ plane. As $|z|$ increases the tilt increases, but $T^{c}$ is never perpendicular to the $z$ plane.

The Szegö projection $\mathbf{S}$ on a domain $\Omega \subset \mathbf{C}^{n}$ is the orthogonal projection from $L^{2}(\partial \Omega)$ to the Hardy space $H^{2}(\Omega)$ of holomorphic functions on $\Omega$ with $L^{2}$ boundary values. The Szegö kernel $S(\zeta, \omega)$ is the kernel for which

$$
\mathbf{S} f(\zeta)=\int_{\partial \Omega} f(\omega) S(\zeta, \omega) d V(\omega) .
$$

See [S2 or $\mathbf{K r}$ ] for discussions of the Szegö projection and the related Bergman projection. We take $d V(\zeta)=d V(x, y, t)=d x d y d t$. This measure is Lebesgue measure on the parameter space $\mathbf{R}^{3}$, not Hausdorff measure on the set $\partial H_{k} \subset \mathbf{C}^{2}$. We define $L^{2}\left(\partial H_{k}\right)$ to be the space of functions $L^{2}$ relative to this measure and we define the Hardy space

$$
H^{2}\left(H_{k}\right)=\left\{f \text { holomorphic on }\left.H_{k}\left|\sup _{\rho>0} \int_{\operatorname{Im} z_{2}=\left|z_{1}\right|^{2 k}+\rho}\right| f\right|^{2} d V<\infty\right\} .
$$

Greiner and Stein [GS] have computed the Szegö kernel for $H_{k}$ with Lebesgue measure on the parameter space. Their formula is

$$
\begin{aligned}
S(\zeta, \omega)=\frac{1}{4 \pi^{2}}\left[\left(\left(\frac{i}{2}[s\right.\right.\right. & \left.\left.-t]+\frac{\left|z_{1}\right|^{2 k}+\left|w_{1}\right|^{2 k}}{2}+\frac{\mu+\eta}{2}\right)^{1 / k}-z_{1} \bar{w}_{1}\right)^{2} \\
& \left.\times\left(\frac{i}{2}[s-t]+\frac{\left|z_{1}\right|^{2 k}+\left|w_{1}\right|^{2 k}}{2}+\frac{\mu+\eta}{2}\right)^{(k-1) / k}\right]^{-1}
\end{aligned}
$$


where we take the principal branch of the root function. It is not difficult to check that for $\zeta, \omega \in \bar{H}_{k}$ the denominator of the kernel vanishes if and only if $\zeta=\omega \in \partial H_{k}$.

3. The pseudometric. In the next sections we define and analyze a pseudometric globally suited to the complex geometry of $\partial H_{k}$. This pseudometric, arrived at by study of the Szegö kernel, allows the treatment of the Szegö kernel as a singular integral kernel. By a pseudometric we mean a nonnegative real function satisfying the approximate symmetry and triangle inequality of 3.3.5 and 3.3.4 below.

If $\zeta$ is a type $2 k$ point, that is if $z=0$, then the pseudometric we define below is essentially $|w|+|s-t|^{1 / 2 k}$. If $\zeta$ is a type 2 point, that is $z \neq 0$, and $\omega$ is nearby then the pseudometric is essentially $|z-w|+|s-t|^{1 / 2}$. Suppose, however, $\zeta$ is a type 2 point and $\omega$ is far from $\zeta$ relative to the distance between $\zeta$ and the type $2 k$ points. From this perspective $\zeta$ appears to be almost a type $2 k$ point and accordingly the pseudometric becomes a hybrid of the two.

For discussions of other pseudometrics and singular integral operators suited to particular geometries and situations see Folland and Stein [FS], Nagel, Stein and Wainger [NSW1, NSW2], and the book Nagel and Stein [NS].

3.1 Definition of the pseudometric.

3.1.1 DEFINITION.

$$
d(\zeta, \omega)=\left|\left(\frac{i}{2}[s-t]+\frac{|z|^{2 k}+|w|^{2 k}}{2}\right)^{1 / k}-z \bar{w}\right|^{1 / 2} .
$$

In (3.1.1) the $k$ th root is chosen so that its argument lies between $-\pi / 2 k$ and $\pi / 2 k$. This is the same as the $k$ th root that appears in the Szegö kernel.

Throughout $\S 3$ we use the notation

$$
P=\left(\frac{i}{2}[s-t]+\frac{|z|^{2 k}+|w|^{2 k}}{2}\right)^{1 / k}, \quad Q=z \bar{w} .
$$

In $\S 3.3$ we use

$$
R_{\zeta \omega}=P^{k}-Q^{k}
$$

The definition of $d$ becomes

$$
d=|P-Q|^{1 / 2}
$$

The Szegö kernel is simply

$$
S=\frac{1}{4 \pi^{2}} \frac{1}{(P-Q)^{2} P^{k-1}} .
$$

3.2 Analysis of the pseudometric. The formula in (3.1.1) for the pseudometric is clearly derived from the Szegö kernel (2.1.3). Here we make some calculations that will be needed below for showing that (3.3.1) does in fact define a pseudometric and for establishing the required estimates.

3.2.1 Lemma. Given $\zeta$ and $\eta \in \partial H_{k}$, let $d_{1}$ be the infimum of all $\delta$ satisfying

$$
\left|\frac{i}{2}[s-t]+\frac{|z|^{2 k}+|w|^{2 k}}{2}-z^{k} \bar{w}^{k}\right|<\delta^{2}|z w|^{k-1}+\delta^{2 k}
$$


and

$$
|z-w|<\delta
$$

Then $|P| \approx|Q|+d_{1}^{2}$.

Proof. Clearly $|P| \gtrsim|z|^{2}$ and $|P| \gtrsim|w|^{2}$ so $|P| \gtrsim|Q|$, always. If $d_{1}(\zeta, z)=\delta$ then at least one of (3.2.1a) and (3.2.1b) is an equality. If (3.2.1b) is equality then at least one of $|z|$ and $|w|$ is $\gtrsim \delta$ so $|P| \gtrsim|z|^{2}+|w|^{2} \gtrsim \delta^{2}$. If (3.2.1a) is equality at least one of $|P|^{k}$ and $|Q|^{k}=|w z|^{k}$ is $\geq \delta^{2}|Q|^{k-1}+\delta^{2 k}$. Hence, since $|P| \geq|Q|$, we have $|P|^{k} \geq \delta^{2}|Q|^{k-1}+\delta^{2 k} \geqslant \delta^{2 k}$. Thus $|P| \geq \delta^{2}$. Summarizing, we have $|P| \geq|Q|+\delta^{2}$. For the opposite inequality, observe that condition (3.2.1a), implies that

$$
|P|^{k} \leqslant|Q|^{k}+\delta^{2}|Q|^{k-1}+\delta^{2 k} \leqslant \max \left(|Q|^{k}, \delta^{2 k}\right) .
$$

Thus $|P| \leqq|Q|+\delta^{2}$.

3.2.2 LeMMA. With $d_{1}$ as in Lemma 3.2.1, $|P-Q| \approx d_{1}^{2}$.

Proof. Let $C_{k}=\max (3, \csc (\pi / 2 k))$. We consider the cases

$$
|z| \leqslant C_{k} \delta
$$

and

$$
|z|>C_{k} \delta
$$

separately. Within each of these cases there are the two cases in which $d_{1}(\zeta, \omega)=\delta$, namely

$$
\begin{gathered}
\left|P^{k}-Q^{k}\right| \leqslant \delta^{2}|Q|^{k-1}+\delta^{2 k} \text { and } \\
|z-w|=\delta
\end{gathered}
$$

or

$$
\begin{gathered}
\left|P^{k}-Q^{k}\right|=\delta^{2}|Q|^{k-1}+\delta^{2 k} \text { and } \\
|z-w| \leqslant \delta .
\end{gathered}
$$

Case (i). Suppose (3.2.3) and (3.2.5). Using the natural homogeneity we may assume $\delta=1$. The problem then becomes to show that

$$
F(s-t, z, w) \equiv\left|\left(\frac{i}{2}[s-t]+\frac{|z|^{2 k}+|w|^{2 k}}{2}\right)^{1 / k}-z \bar{w}\right|
$$

is bounded uniformly above and below when

$$
\begin{gathered}
\left|\frac{i}{2}[s-t]+\frac{|z|^{2 k}+|w|^{2 k}}{2}-z^{k} \bar{w}^{k}\right| \leqslant|z w|^{k-1}+1, \\
|z-w|=1, \text { and } \\
|z| \leqslant C_{k} .
\end{gathered}
$$

Conditions (3.2.8) imply $|s-t| \leqslant|z|^{2 k}+|w|^{2 k}+|z w|^{k-1}+1 \leqslant C$. This combined with (3.2.8b and c) implies that the set $K$ of $(s-t, z, w) \in \mathbf{R} \times \mathbf{C} \times \mathbf{C}$ satisfying conditions (3.2.8) is compact. As observed in $\$ 2.1$, the function $F$ in (3.2.7) vanishes 
if and only if $s-t=0$ and $z=w$. Therefore, because of $(3.2 .8 \mathrm{~b}), F$ is never zero on $K$. Since $F$ is continuous, we obtain (3.2.7).

Case (ii). Suppose (3.2.3) and (3.2.6). We show that in this case it is true that in fact

$$
\left|P-Q e^{2 \pi i j / k}\right| \approx \delta^{2} \text { for all } j=0,1, \ldots, k-1 .
$$

First observe that conditions (3.2.3) and (3.2.6b) imply

$$
\begin{gathered}
|z| \leq \delta, \quad|w| \leq \delta, \quad|B| \leq \delta^{2}, \quad \text { and } \\
\left|P-Q e^{2 \pi i j / k}\right| \leq \delta^{2} \quad \text { all } j=0,1, \ldots, k-1 .
\end{gathered}
$$

Now, $\prod_{j=0}^{k-1}\left(P-Q e^{2 \pi i j / k}\right)=P^{k}-Q^{k}$. By (3.2.6a) and (3.2.10) $\left|P^{k}-Q^{k}\right| \approx \delta^{2 k}$. Thus

$$
\prod_{j=0}^{k-1}\left|P-Q e^{2 \pi i j / k}\right| \approx \delta^{2 k}
$$

Combining this with (3.2.11) we deduce (3.2.9). In particular, taking $j=0,|P-Q|$ $\approx \delta^{2}$.

Case (iii). Suppose (3.2.4) and (3.2.5). We show that these conditions imply (3.2.6) with $=$ replaced by $\approx$. This reduces case (iii) to case (iv). First,

$$
\left|P^{k}-Q^{k}\right| \geqslant\left|\operatorname{Re}\left(P^{k}-Q^{k}\right)\right|=\frac{1}{2}\left|z^{k}-w^{k}\right|^{2}=\frac{1}{2}|z-w|^{2} \prod_{j=1}^{k-1}\left|z-w e^{2 \pi i j / k}\right|^{2} .
$$

By (3.2.5b) $|z-w|^{2} \approx \delta^{2}$. For the other factors, note that the condition in (3.2.4) that $|z|>(\csc (\pi / 2 k)) \delta$ and condition $(3.2 .5 b)$ (here we need only $\leqslant$ ) imply

$$
|\arg z \bar{w}|<\pi / 2 k \text {. }
$$

Thus

The angle between $z$ and $w e^{2 \pi i j / k}, j=1,2, \ldots, k-1$, is more than $\pi / 2 k$.

The condition in (3.2.4) that $|z|>3 \delta$ and condition (3.2.5b) (again, we need only $\leqslant)$ imply

$$
|z| \approx|w| \geq \delta \quad \text { and } \quad|Q| \geq \delta^{2}
$$

By (3.2.14) and (3.2.15)

$$
\left|z-w e^{2 \pi i j / k}\right|^{2} \geq|z|^{2} \approx|z w| \text { for } j=1,2, \ldots, k-1 .
$$

Combining (3.2.16) and condition (3.2.5b) with (3.2.12) above we have $\left|P^{k}-Q^{k}\right| \gtrsim$ $\delta^{2}|z w|^{k-1}$. Because of (3.2.15), $\left|P^{k}-Q^{k}\right| \geq \delta^{2}|z w|^{k-1}+\delta^{2 k}$. Since condition (3.2.5a) is the opposite inequality, we have $\left|P^{k}-Q^{k}\right| \approx \delta^{2}|z w|^{k-1}+\delta^{2}$, as claimed.

Case (iv). Suppose (3.2.4) and (3.2.6). We use the formula

$$
P-Q=\left(P^{k}-Q^{k}\right) / \prod_{j=1}^{k-1}\left(P-Q e^{2 \pi i j / k}\right) .
$$


Conditions (3.2.4) and (3.2.6b) imply $|w| \geq \delta$ and

$$
|Q| \geq \delta^{2} \text {. }
$$

By Lemma 3.2.1 $|P| \approx|Q|+\delta^{2}$ so, with (3.2.18),

$$
|P| \approx|Q| \text {. }
$$

For the numerator in (3.2.17), note that condition (3.2.6a) and (3.2.18) give

$$
\left|P^{k}-Q^{k}\right| \approx \delta^{2}|Q|^{k-1}+\delta^{2 k} \approx \delta^{2}|Q|^{k-1} \text {. }
$$

For the denominator in (3.2.17) observe that, as in case (iii),the condition in (3.2.4) that $|z|>(\csc |\pi / 2 k|) \delta$ and condition (3.2.6b) imply $|\arg z \bar{w}|<\pi / 2 k$. Since $P$ is a $k$ th root taken with $|\arg P|<\pi / k$, the angle between $P$ and $Q e^{2 \pi i j / k}=z \bar{w} e^{2 \pi i j / k}$ is larger than $\pi / 2 k$ for $j=1, \ldots, k-1$. Therefore, because of equation (3.2.19), $\left|P-Q e^{2 \pi i j / k}\right| \approx|Q|, j=1, \ldots, k-1$, and

$$
\prod_{j=1}^{k-1}\left|P-Q e^{2 \pi i j / k}\right| \approx|Q|^{k-1}
$$

Combining this estimate for the denominator with (3.2.20) for the numerator in (3.2.17) we obtain

$$
|P-Q| \approx \delta^{2}|P|^{k-1} /|Q|^{k-1} \approx \delta^{2} .
$$

3.2.21 Proposition. $d \approx d_{1}$, where $d_{1}$ is as in Lemma 3.2.1.

Proof. Since $d=|P-Q|^{1 / 2}$ this is simply a restatement of Lemma 3.2.2.

Lemmas 3.2.1 and 3.2.2 will be seen later to contain also the basic size estimate on the Szegö kernel.

Remark. The $d_{1}$ defined in Lemma 3.2.1 is similar to the pseudometric $\rho_{6}$ of Nagel, Stein, and Wainger [NSW1], which is given in terms of a defining function for $H_{k}$ and Kohn's lambda function [Ko]. The right side of (3.2.1a) is essentially Kohn's lambda function for $H_{k}$. Our definition of $d_{1}$ becomes a definition for $\rho_{6}$ if in (3.2.1a) we replace the $|z w|^{k-1}$ with $|z|^{2 k-2}$ and we add to (3.2.1b) the conditions $|s-t|<\delta$ and $\left.|| z\right|^{2 k}-|w|^{2 k} \mid<\delta$. The Nagel-Stein-Wainger [NSW1] definition was designed for local use and is equivalent to the pseudometric here only on compact sets, even in the strongly pseudoconvex case $k=1$.

The condition contributed by the real part in the left side of (3.2.2a) is redundant. Also, in the right side of (3.2.2a) we may replace the expression $|z w|$ by $|z|^{2}$ or by $|w|^{2}$. The resulting definition is useful for the volume calculation we make. We record it in the following.

3.2.22 Proposition. Given $\zeta$ and $\eta \in \partial H_{k}$, let $d_{2}$ be the infimum of all $\delta$ satisying

$$
\left|\frac{1}{2}[s-t]+\operatorname{Im}\left(\bar{z}^{k} w^{k}\right)\right|<\delta^{2}|z|^{2 k-2}+\delta^{2 k}
$$

and

(3.2.22b)

$$
|z-w|<\delta .
$$

Then $d_{1} \approx d_{2}$ where $d_{1}$ is as in Lemma 3.2.1.

Proof.

$$
\operatorname{Im}\left(P^{k}-Q^{k}\right)=\frac{1}{2}[s-t]+\operatorname{Im}\left(\bar{z}^{k} w^{k}\right)
$$


so clearly $d_{1} \geqslant d_{2}$. For the other direction observe that (3.2.22b) implies

$$
\begin{aligned}
\operatorname{Re}\left(P^{k}-Q^{k}\right) & =\frac{1}{2}\left|z^{k}-w^{k}\right|^{2} \leqslant|z-w|^{2}\left(|z|^{2(k-1)}+|w|^{2(k-1)}\right) \\
& \leq \delta^{2}\left(|z|^{2(k-1)}+|w|^{2(k-1)}\right) \leq \delta^{2}|z w|^{k-1}+\delta^{2 k} \\
& \leq \delta^{2}|z|^{2 k-2}+\delta^{2 k} .
\end{aligned}
$$

Condition (3.2.22a) is the corresponding inequality for $\operatorname{Im}\left(P^{k}-Q^{k}\right)$ (recall (3.2.23)). Therefore the pair (3.2.22b) and (3.2.22a) imply the pair (3.2.1b) and (3.2.1a) with $\leqslant$ in the second pair. Thus $d_{1} \leqslant d_{2}$.

For use in $\S 6$ we record the following variation as well.

3.2.24 Proposition. Given $\zeta$ and $\eta \in \partial H_{k}$, let $d_{3}$ be the infimum of all $\delta$ satisfying

$$
\left|\frac{i}{2}[s-t]+\frac{|z|^{2 k}+|w|^{2 k}}{2}-z^{k} \bar{w}^{k}\right|<\frac{1}{4}\left(\sin \frac{\pi}{k}\right)^{2 k-2}|z|^{2 k-2} \delta^{2}+\frac{1}{2} \delta^{2 k}
$$

and

$$
|z-w|<\delta
$$

Then $d_{3} \approx d_{1}$ where $d_{1}$ is as in Lemma 3.2.1.

In (3.2.24) the constants $\frac{1}{4}\left(\sin (\pi / k)^{2 k-2}\right.$ and $\frac{1}{2}$ may be replaced by other positive constants. The choice made here will be useful in $\S 6$. Note that we have established $d \approx d_{1} \approx d_{2} \approx d_{3}$.

3.3 Triangle inequality, symmetry, and homogeneity. In view of the equivalence shown in the preceding section, an inequality $d(\zeta, \omega) \leqq d(\zeta, \nu)+d(\nu, \omega)$ for $d_{1}$ implies the same inequality (with possibly different constants) for $d$.

3.3.1 Lemma. $d_{1}(\zeta, \omega) \lesssim d_{1}(\zeta, \nu)+d_{1}(\nu, \omega)$.

Proof. It suffices to show that if

$$
d_{1}(\zeta, \nu) \leqslant \delta \quad \text { and } \quad d_{1}(\nu, \omega) \leqslant \delta
$$

then

$$
d_{1}(\zeta, \omega) \leq \delta .
$$

In the notation (3.1.2b) and with $\zeta=(z, t), \nu=(u, r), \omega=(w, s)$, the assumptions are

$$
\left|R_{\zeta \nu}\right|<\delta^{2}|z u|^{k-1}+\delta^{2 k}, \quad|z-u|<\delta
$$

and

$$
\left|R_{\nu \omega}\right|<\delta^{2}|u w|^{k-1}+\delta^{2 k}, \quad|u-w|<\delta .
$$

These clearly imply, by the Euclidean triangle inequality, $|z-w| \leq \delta$. For the other requirement of (3.3.3),

$$
\begin{aligned}
\left|R_{\zeta \omega}\right|= & \left|R_{\zeta \nu}+R_{\nu \omega}+\left(z^{k}-u^{k}\right)\left(\bar{u}^{k}-\bar{w}^{k}\right)\right| \\
\leq & \left(\delta^{2}|z u|^{k-1}+\delta^{2 k}\right)+\left(\delta^{2}|u w|^{k-1}+\delta^{2 k}\right) \\
& +\delta^{2}\left(|z u|^{k-1}+|z w|^{k-1}+|u w|^{k-1}+|u \bar{u}|^{k-1}\right) .
\end{aligned}
$$


If $|w|>3 \delta$ then $|w| \approx|z| \approx|u|$ and $\left|R_{\zeta \omega}\right| \lesssim \delta^{2}|z w|^{k-1}+\delta^{2 k}$. If $|w| \lesssim 3 \delta$ then $|z| \leq \delta$ and $|u| \leq \delta$ also, so

$$
\left|R_{\zeta \omega}\right| \leq \delta^{2 k} \approx \delta^{2}|z w|^{k-1}+\delta^{2 k}
$$

as needed.

3.3.4 Proposition (Triangle inequality). $d(\zeta, \omega) \lessgtr d(\zeta, \nu)+d(\nu, \omega)$.

3.3.5 Proposition (Symmetry). $d(\zeta, \omega) \approx d(\omega, \zeta)$.

Proof. This is clear.

With Propositions 3.3.4 and 3.3.5 we are now justified in referring to $d$ as a pseudometric.

3.3.6 Proposition (Homogeneity).

$$
\text { Let } \tau \zeta=\left(\tau z, \tau^{2 k} t\right) \text { and } \tau \omega=\left(\tau w, \tau^{2 k} s\right) .
$$

Then $d(\tau \zeta, \tau \omega)=\tau d(\zeta, \omega)$.

Proof. The homogeneity is clear from the definitions.

3.4 Volumes of balls.

3.4.1 Definition. $B_{\zeta}(\delta) \equiv B_{\zeta}^{d}(\delta) \equiv\left\{\nu \in \partial H_{k}: d(\zeta, \nu)<\delta\right\} . B_{\zeta}(\delta)$ is the open ball of $d$-radius $\delta$ centered at $\zeta$. Recall that the volume we use is Lebesgue measure on the parameter space $\mathbf{R}^{3}$.

3.4.2 LEMMA.

$$
\operatorname{Vol}\left[B_{\zeta}^{d_{2}}(\delta)\right]=4 \pi \delta^{2}\left(\frac{(\sin (\pi / k))^{2 k-2}}{4}|z|^{2 k-2} \delta^{2}+\frac{1}{2} \delta^{2 k}\right)
$$

Proof. By Cavalieri's principle, the volume is the area of the disc $|z-w|<\delta$, which is $\pi \delta^{2}$, multiplied by the height

$$
4\left(\frac{(\sin (\pi / k))^{2 k-2}}{4}|z|^{2 k-2} \delta^{2}+\frac{1}{2} \delta^{2 k}\right) .
$$

3.4.3 Lemma. For all constants $C \geqslant 0$,

$$
\operatorname{Vol}\left[B_{\zeta}^{d_{2}}(C \delta)\right] \leqslant\left[\max \left(C^{4}, C^{2(k+1)}\right)\right] \operatorname{Vol}\left[B_{\zeta}^{d^{2}}(\delta)\right]
$$

ProOF. This follows from the expression given in Lemma 3.4.2 for volume.

3.4.4 Proposition. $\operatorname{Vol}\left[B_{\zeta}^{d}(\delta)\right] \approx \delta^{2}\left(\delta^{2}|z|^{2 k-2}+\delta^{2 k}\right)$.

Proof. By Propositions 3.2.22 and 3.2.21 there exist constants $C_{1}$ and $C_{2}$ such that

$$
B_{\zeta}^{d_{2}}\left(C_{1} \delta\right) \subset B_{\zeta}^{d}(\delta) \subset\left(B_{\zeta}^{d_{2}}\left(C_{2} \delta\right)\right), \text { for all } \zeta \text { and all } \delta \text {. }
$$

Now use Lemmas 3.4.2 and 3.4.3.

3.4.5 Proposition (Doubling PROPERTy). For each constant $C_{3}$ there exists a constant $C_{4}$ such that

$$
\operatorname{Vol}\left[B_{\zeta}\left(C_{3} \delta\right)\right] \leqslant C_{4} \operatorname{Vol}\left[B_{\zeta}(\delta)\right]
$$


Proof. This follows immediately from Proposition 3.4.4.

Proposition 3.4.5 gives an upper bound on the volume of expanded balls but gives no useful information on a lower bound. There is, however, lower bound information derivable from the expression for volume given in Proposition 3.4.4. We do not use the following proposition or its corollary except for completeness in the integral formulae in the next section. The corollary allows us to obtain equivalence rather than just upper bounds in the formulae. This allows us to see, for instance, that the Szegö kernel is in fact not absolutely integrable.

3.4.6 Proposition. Let $C_{5}$ and $C_{6}$ be constants (guaranteed to exist by Proposition 3.4.4) such that

$$
C_{5}\left[\delta^{2}\left(\delta^{2}|z|^{2 k-2}+\delta^{2 k}\right)\right] \leqslant \operatorname{Vol}\left[B_{\zeta}^{d}(\delta)\right] \leqslant C_{6}\left[\delta^{2}\left(\delta^{2}|z|^{2 k-1}+\delta^{2 k}\right)\right] .
$$

Then

$$
\operatorname{Vol}\left[B_{\zeta}^{d}(C \delta)\right] \geqslant C^{4}\left(C_{5} / C_{6}\right) \operatorname{Vol}\left[B_{\zeta}(\delta)\right] \quad \text { for all } C \geqslant 1
$$

ProOF.

$$
\begin{aligned}
\operatorname{Vol}\left[B_{\zeta}^{d}(C \delta)\right] & \geqslant C_{5}(C \delta)^{2}\left((C \delta)^{2}|z|^{2 k-2}+(C \delta)^{2 k}\right) \\
& \geqslant C_{5} C^{4} \delta^{2}\left(\delta^{2}|z|^{2 k-2}+\delta^{2 k}\right) \geqslant\left(C_{5} C^{4} / C_{6}\right) \operatorname{Vol}\left[B_{\zeta}^{d}(\delta)\right]
\end{aligned}
$$

3.4.7 Corollary. If $C^{4} \geqslant 2 C_{6} / C_{5}$, where $C_{5}$ and $C_{6}$ are the constants in Proposition 3.4.6, then

$$
\operatorname{Vol}\left[B_{\zeta}(C \delta) \backslash B_{\zeta}(\delta)\right] \geqslant \operatorname{Vol}\left[B_{\zeta}(\delta)\right]
$$

Proof. This follows immediately from Proposition 3.4.6.

Next we show that an $\varepsilon$ fringe on a ball of radius $R$ becomes negligible relative to the whole ball as $R$ becomes large. We require an observation about homogeneity. Recall that we use the dilation $\tau \zeta=\left(\tau z, \tau^{2 k} t\right), \tau>0$, of (3.3.7).

3.4.8 LEMMA.

$$
\tau \omega \in B_{\tau \zeta}(\tau \delta) \quad \text { if and only if } \quad \omega \in B_{\zeta}(\delta)
$$

and

$$
\operatorname{Vol}\left[B_{\tau \zeta}(\tau \delta)\right]=\tau^{2 k+2} \operatorname{Vol}\left[B_{\zeta}(\tau)\right]
$$

Proof. (3.4.9) follows from the homogeneity (3.3.6) of the pseudometrics. (3.4.10) follows from (3.4.9) and the fact that volume is simply Lebesgue measure on $\mathbf{R}^{3}$.

3.4.11 Proposition. Fix $\varepsilon \in R$ and $\zeta \in \partial H_{k}$. Then

$$
\lim _{R \rightarrow \infty} \frac{\operatorname{Vol}\left[B_{\zeta}(R) \backslash B_{\zeta}(R-\varepsilon)\right]}{\operatorname{Vol}\left[B_{\zeta}(R)\right]}=0 .
$$


Proof. By Lemma 3.4.8 with $\tau=1 / R$,

$$
\begin{aligned}
\frac{\operatorname{Vol}\left[B_{\zeta}(R) \backslash B_{\zeta}(R-\varepsilon)\right]}{\operatorname{Vol}\left[B_{\zeta}(R)\right]} & =\frac{R^{-2 k-2} \operatorname{Vol}\left[B_{\zeta / R}(1) \backslash B_{\zeta / R}(1-\varepsilon / R)\right]}{R^{-2 k-2} \operatorname{Vol}\left[B_{\zeta / R}(1)\right]} \\
& =\frac{\operatorname{Vol}\left[B_{\zeta / R}(1) \backslash B_{\zeta / R}(1-\varepsilon / R)\right]}{\operatorname{Vol}\left[B_{\zeta / R}(1)\right]} .
\end{aligned}
$$

By the formula for volume in Proposition 3.4.4,

$$
\operatorname{Vol}\left[B_{\zeta / R}(1)\right] \approx|\zeta / R|^{2 k-2}+1 \geqslant C .
$$

Therefore,

$$
\frac{\operatorname{Vol}\left[B_{\zeta}(R) \backslash B_{\zeta}(R-\varepsilon)\right]}{\operatorname{Vol}\left[B_{\zeta}(R)\right]} \leqslant C^{-1} \operatorname{Vol}\left[B_{\zeta / R}(1) \backslash B_{\zeta / R}\left(1-\frac{\varepsilon}{R}\right)\right] .
$$

Letting $R$ tend to $\infty$, the right-hand side tends to zero. Hence the left-hand side tends to zero.

3.5 Integral formulae.

\subsubsection{Proposition.}

$$
\int_{\omega \notin B_{\zeta}(\delta)} \frac{d V(\omega)}{\operatorname{Vol}\left[B_{\zeta}(d(\zeta, \omega))\right][d(\zeta, \omega)]^{\alpha}} \approx \begin{cases}1 / \delta^{\alpha}, & \alpha>0, \\ \text { diverges, } & \alpha \leqslant 0 .\end{cases}
$$

Proof. Choose $C$ satisfying the hypothesis of Corollary 3.4.7. Observe that $C_{6} \geqslant C_{5}$ so $C \geqslant 2>1$. Now,

$$
\begin{aligned}
& \int_{\omega \notin B_{\zeta}(\delta)} \frac{d V(\omega)}{\operatorname{Vol}\left[B_{\zeta}(d(\zeta, \omega))\right][d(\zeta, \omega)]^{\alpha}} \\
&=\sum_{i=0}^{\infty} \int_{B_{\zeta}\left(C^{i+1} \delta\right) \backslash B_{\zeta}\left(C^{i} \delta\right)} \frac{d V(\omega)}{\operatorname{Vol}\left[B_{\zeta}(d(\zeta, \omega))\right][d(\zeta, \omega)]^{\alpha}} \\
& \approx \sum_{i=0}^{\infty} \frac{\operatorname{Vol}\left[B_{\zeta}\left(C^{i+1} \delta\right) \backslash B_{\zeta}\left(C^{i} \delta\right)\right]}{\operatorname{Vol}\left[B_{\zeta}(d(\zeta, \omega))\right]\left(C^{i} \delta\right)^{\alpha}} \\
& \approx \sum_{i=0}^{\infty} \frac{\operatorname{Vol}\left[B_{\zeta}\left(C^{i} \delta\right)\right]}{\operatorname{Vol}\left[B_{\zeta}\left(C^{i} \delta\right)\right]\left(C^{i} \delta\right)^{\alpha}}=\frac{1}{\delta^{\alpha}} \sum_{i=0}^{\infty} \frac{1}{\left(C^{\alpha}\right)^{i}} \\
& \approx\left\{\begin{array}{l}
1 / \delta^{\alpha}, \quad \alpha>0, \\
\text { diverges, }
\end{array} \quad \alpha \leqslant 0 . \quad \square\right.
\end{aligned}
$$

Next we integrate inside the ball with powers of $d$ in the numerator.

3.5.2 Proposition.

$$
\int_{\omega \in B_{\zeta}(\delta)} \frac{[d(\zeta, \omega)]^{\alpha} d V(\omega)}{\operatorname{Vol}\left[B_{\zeta}(d(\zeta, \omega))\right]} \approx \begin{cases}\delta^{\alpha}, & \alpha>0, \\ \text { diverges, } & \alpha \leqslant 0 .\end{cases}
$$

Proof. The proof is similar to that of Proposition 3.5.1. 
Here, we integrate on the $\varepsilon$ fringe of a ball of radius $R$.

\subsubsection{Proposition.}

$$
\lim _{R \rightarrow \infty} \int_{\omega \in B_{\zeta}(R) \backslash B_{\zeta}(R-\varepsilon)} \frac{1}{\operatorname{Vol}\left[B_{\zeta}(d(\zeta, \omega))\right]} d V(\omega)=0 .
$$

Proof. The absolute value of the integral is dominated by

$$
C \frac{\operatorname{Vol}\left[B_{\zeta}(R) \backslash B_{\zeta}(R-\varepsilon)\right]}{\operatorname{Vol}\left[B_{\zeta}(R)\right]} .
$$

Hence, by Proposition 3.4.11, the limit as $R \rightarrow \infty$ is zero.

4. Connecting curves. In the next sections we show that the points that lie a distance $\delta$ away from $\zeta$ are the points that can be reached from $\zeta$ along curves whose tangents always lie in the complex tangent space and whose projections in the $z$ plane have Euclidean length no more than $\delta$. We use this characterization of our balls in $\$ 6.2$ in the proof of the Calderón-Zygmund condition for the Szegö kernel.

4.1 Curves and lifts. Recall (\$2) that the tangent space to a hypersurface in $\mathbf{C}^{n}$ possesses an even dimensional subspace we call the complex tangent space $T^{c}$. At any point $\zeta \in \partial H_{k}, T^{c}$ is the plane spanned by

$$
\operatorname{Re} Z=\left(1,0,2 k\left(x^{2}+y^{2}\right)^{k-1} y\right) \text { and } \operatorname{Im} Z=\left(0,-1,2 k\left(x^{2}+y^{2}\right)^{k-1} x\right)
$$

where $\zeta=(z, t)=(x, y, t)$ and the tangent space to $\partial H_{k}$ has been identified with $\mathbf{R}^{3}$. Since the complex tangent space to $\partial H_{k}$ is never perpendicular to the $z$ plane, we have the following.

\subsubsection{Proposition.}

A. If $\tilde{\Upsilon}:[a, b] \rightarrow \partial H_{k}$ is any piecewise differentiable curve in $\partial H_{k}$ whose tangent always lies in $T^{c}$ then

$$
t(\tau)=-2 k \int_{0}^{\tau} \rho^{2 k}(\sigma) \frac{d \theta(\sigma)}{d \sigma} d \sigma+t_{0}, \quad a \leqslant \tau \leqslant b,
$$

where $\tilde{\Upsilon}=(x(\tau), y(\tau), t(\tau)),(\rho, \theta)$ is $(x, y)$ in polar coordinates, and $t_{0}=t(0)$.

B. (Lifting) Given any piecewise differentiable curve $\Upsilon:[a, b] \rightarrow \mathbf{C}$ whose image lies in the $(x, y)$ plane and given any height $t_{0}$, there is a unique lifted curve $\tilde{\Upsilon}$ : $[a, b] \rightarrow \partial H_{k}$ in $\partial H_{k}$ whose tangent always lies in $T^{c}$, whose projection in the $(x, y)$ plane is $\Upsilon$, and whose initial height is $t_{0}$.

Proof. If $\dot{\tilde{\Upsilon}}$ always lies in $T^{c}$ then, using (4.1.1), there exist coefficient functions $\lambda(\tau)$ and $\mu(\tau)$ such that

$$
\begin{aligned}
&(\dot{x}(\tau), \dot{y}(\tau), \dot{t}(\tau))=\lambda(\tau) \operatorname{Re} Z_{\Upsilon(\tau)}+\mu(\tau) \operatorname{lm} Z_{\Upsilon(\tau)} \\
& \quad=\left(\lambda(\tau),-\mu(\tau), 2 k\left(x^{2}(\tau)+y^{2}(\tau)\right)^{k-1}[\lambda(\tau) y(\tau)+\mu(\tau) x(\tau)]\right) .
\end{aligned}
$$


Here, dot denotes $\tau$ differentiation. We see that $\lambda(\tau)=\dot{x}(\tau), \mu(\tau)=-\dot{y}(\tau)$ and that therefore

$$
\begin{aligned}
\dot{t}(\tau) & =2 k\left(x^{2}(\tau)+y^{2}(\tau)\right)^{k-1}[\dot{x}(\tau) y(\tau)-\dot{y}(\tau) x(\tau)] \\
& =-2 k \rho^{2 k}(\tau) \dot{\theta}(\tau) .
\end{aligned}
$$

Integration gives (4.1.3). For part $\mathrm{B}$, simply note that given the curve $\Upsilon$ and the intial height $t_{0},(4.1 .3)$ defines $\tilde{\Upsilon}$.

4.2 The pseudometric in terms of curves whose tangents lie in the complex tangent space. We use the conventions that $\Upsilon$ is the projection of $\tilde{\Upsilon}$ into the $z$ plane and that $L(\Upsilon)$ is the Euclidean length of $\Upsilon$.

4.2.1 LEMmA. If the points $\zeta \in \partial H_{k}$ and $\omega \in \partial H_{k}$ can be connected by a curve $\tilde{\Upsilon}$ with $\dot{\tilde{\Upsilon}} \in T^{c}$ and $L(\Upsilon) \leqslant \delta$, then $d(\zeta, \omega) \leqslant C \delta$, where $d$ is the pseudometric defined in $\S 3$ and $C$ is a constant that depends only on $k$.

Proof. Let $\tilde{\Upsilon}:[a, b] \rightarrow \partial H_{k}$ be as described with $\tilde{\Upsilon}(a)=\zeta$ and $\tilde{\Upsilon}(b)=\omega$. To show that $d(\zeta, \omega)<C \delta$ we choose to work with $d_{2}$ defined in (3.2.22). Clearly the condition $L(\Upsilon) \leqslant \delta$ implies (3.2.22b). To show (3.2.22a) we use the polar coordinates $z=\rho_{a} e^{i \theta_{a}}$ and $w=\rho_{b} e^{i \theta_{b}}$ to rewrite (3.2.22a) as

$$
\left|\frac{1}{2}[s-t]+\rho_{a}^{k} \rho_{b}^{k} \sin \left[k\left(\theta_{b}-\theta_{a}\right)\right]\right|<\delta^{2} \rho_{a}^{2(k-1)}+\delta^{2 k} .
$$

By Proposition 4.1.2, the assumption $\dot{\tilde{\Upsilon}} \in T^{c}$ gives

$$
s-t=-2 k \int_{a}^{b} \rho^{2 k}(\sigma) \dot{\theta}(\sigma) d \sigma,
$$

where $(\rho(\tau), \theta(\tau)), \tau \in[a, b]$, is $\Upsilon$ written in polar form. Using (4.2.3), (4.2.2) becomes

$$
\left|\rho_{a}^{k} \rho_{b}^{k} \sin \left[k\left(\theta_{b}-\theta_{a}\right)\right]-k \int_{a}^{b} \rho^{2 k}(\sigma) \dot{\theta}(\sigma) d \sigma\right|<\delta^{2} \rho_{a}^{2(k-1)}+\delta^{2 k} .
$$

The assumption on the length of $\Upsilon$ is that

$$
L(\Upsilon) \leqslant \delta
$$

whence

$$
\int_{a}^{b} \rho(\sigma)|\dot{\theta}(\sigma)| d \sigma \leqslant \delta .
$$

We show that (4.2.5) implies (4.2.4). The cases of $\rho$ small and $\rho$ large are treated separately.

Case (i). Suppose $\rho_{a} \leqslant 2 \delta$. Then by (4.2.5a), $|\rho(\tau)| \leqslant 3 \delta$ for all $\tau \in[a, b]$. First, this implies

$$
\left|\rho_{a}^{k} \rho_{b}^{k} \sin \left[\left(\theta_{b}-\theta_{a}\right)\right]\right| \leq \delta^{2 k} .
$$

Also, with (4.2.5b),

$$
\left|k \int_{a}^{b} \rho^{2 k}(\sigma) \dot{\theta}(\sigma) d \sigma\right| \leq \delta^{2 k-1} \int_{a}^{b} \rho(\sigma)|\dot{\theta}(\sigma)| d \sigma \lesssim \delta^{2 k} .
$$

Hence (4.2.4) holds. 
Case (ii). Suppose $\rho_{a}>2 \delta$. Then by (4.2.5a) we can choose $R$ and $r$ with

$$
r \leqslant \rho(\tau) \leqslant R \text { for all } \tau \in[a, b] \text { and }
$$

$$
R \approx r \approx \rho(\tau) \text { all } \tau \in[a, b] \text { and }|R-r|<2 \delta .
$$

For convenience we take $\theta_{b}-\theta_{a} \geqslant 0$. Since $\theta-\theta^{3} / 6 \leqslant \sin \theta \leqslant \theta$ for all $\theta \geqslant 0$ we have then, with (4.2.6),

$$
r^{2 k} k\left(\theta_{b}-\theta_{a}\right)-\frac{r^{2 k}\left[k\left(\theta_{b}-\theta_{a}\right)\right]^{3}}{6} \leqslant \rho_{a}^{k} \rho_{b}^{k} \sin \left[k\left(\theta_{b}-\theta_{a}\right)\right] \leqslant R^{2 k} k\left(\theta_{b}-\theta_{a}\right) .
$$

Define $\dot{\theta}_{+}=\frac{1}{2}(|\dot{\theta}|+\dot{\theta})$ and $\dot{\theta}_{-}=\frac{1}{2}(|\dot{\theta}|-\dot{\theta})$ to be the positive and negative parts, respectively, of $\dot{\theta}$. Then, for the second term on the left side of (4.2.4) we have, using (4.2.6),

$$
\begin{aligned}
\int_{a}^{b} \rho^{2 k}(\sigma) \dot{\theta}(\sigma) d \sigma & =\int_{a}^{b} \rho^{2 k}(\sigma) \dot{\theta}_{+}(\sigma) d \sigma-\int_{a}^{b} \rho^{2 k}(\sigma) \dot{\theta}_{-}(\sigma) d \sigma \\
& \leqslant R^{2 k} \int_{a}^{b} \dot{\theta}_{+}(\sigma) d \sigma-r^{2 k} \int_{a}^{b} \dot{\theta}_{-}(\sigma) d \sigma \\
& =R^{2 k} \int_{a}^{b} \dot{\theta}(\sigma) d \sigma+\left(R^{2 k}-r^{2 k}\right) \int_{a}^{b} \dot{\theta}_{-}(\sigma) d \sigma \\
& =R^{2 k}\left(\theta_{b}-\theta_{a}\right)+\left(R^{2 k}-r^{2 k}\right) \int_{a}^{b} \dot{\theta}_{-}(\sigma) d \sigma
\end{aligned}
$$

and similarly

$$
\int_{a}^{b} \rho^{2 k}(\sigma) \dot{\theta}(\sigma) d \sigma \geqslant r^{2 k}\left(\theta_{b}-\theta_{a}\right)-\left(R^{2 k}-r^{2 k}\right) \int_{a}^{b} \dot{\theta}_{-}(\sigma) .
$$

Combining (4.2.8) with (4.2.9) and with (4.2.10), we have

$$
\begin{aligned}
& \rho_{a}^{k} \rho_{b}^{k} \sin \left[k\left(\theta_{b}-\theta_{a}\right)\right]-k \int_{a}^{b} \rho^{2 k}(\sigma) \dot{\theta}(\sigma) d \sigma \\
& \quad \leqslant\left(R^{2 k}-r^{2 k}\right) k\left[\left(\theta_{b}-\theta_{a}\right)+\int_{a}^{b} \dot{\theta}_{-}(\sigma) d \sigma\right] .
\end{aligned}
$$

(4.2.12) $\rho_{a}^{k} \rho_{a}^{k} \sin \left[k\left(\theta_{b}-\theta_{a}\right)\right]-k \int_{a}^{b} \rho^{2 k}(\sigma) \dot{\theta}(\sigma) d \sigma$

$$
\geqslant\left(r^{2 k}-R^{2 k}\right) k\left[\left(\theta_{b}-\theta_{a}\right)+\int_{a}^{b} \dot{\theta}_{-}(\sigma) d \sigma\right]-\frac{r^{2 k}\left[k\left(\theta_{b}-\theta_{a}\right)\right]^{3}}{6} .
$$

Now, (4.2.6) and (4.2.5b) imply

$$
\int_{a}^{b}|\dot{\theta}(\sigma)| d \sigma \leqslant \frac{1}{R} \int_{a}^{b} \rho(\sigma)|\dot{\theta}(\sigma)| d \sigma \leqslant \frac{\delta}{R} .
$$

Hence

$$
\left|\theta_{b}-\theta_{a}\right| \leqslant \frac{\delta}{R} \quad \text { and } \quad \int_{a}^{b} \dot{\theta}_{-}(\sigma) d \sigma \leqslant \frac{\delta}{R}
$$


Using (4.2.13) and (4.2.7) we find that the right side of (4.2.11) is

$$
\begin{aligned}
& \lesssim\left(R^{2 k}-r^{2 k}\right) \frac{\delta}{R} \lesssim R^{2 k-1}(R-r) \frac{\delta}{R} \lesssim R^{2 k-2} \delta^{2} \\
& \approx \rho_{a}^{2(k-1)} \delta^{2} \approx \rho_{a}^{2(k-1)} \delta^{2}+\delta^{2 k} .
\end{aligned}
$$

We obtain the same estimate on the absolute value of the right side of (4.2.12) by noting that the extra cubic term is $\leqslant R^{2 k} \delta^{3} / R^{3} \leq R^{2 k-2} \delta^{2}$. Together these estimates give (4.2.4).

The following two lemmas give the converse to Lemma 4.2.1.

4.2.14 LEMmA. If $\zeta=(z, t)$ and $\zeta^{\prime}=\left(z, t^{\prime}\right)$ have the same first coordinate, then $\zeta$ and $\zeta^{\prime}$ can be connected by a curve $\tilde{\Upsilon}$ with $\tilde{\Upsilon} \in T^{c}$ and $L(\Upsilon) \leqslant C d\left(\zeta, \zeta^{\prime}\right)$, where $C$ is a constant that depends only on $k$.

Proof. We exhibit such a curve. Let $z=r e^{i \theta}$. Let $\Upsilon$ be the curve in the plane that goes from $r e^{i \theta}$ to $(r+\Delta r) e^{i \theta}$ along a straight line, goes from $(r+\Delta r) e^{i \theta}$ to $(r+\Delta r) e^{i(\theta+\Delta \theta)}$ along the circle $|\zeta|=r+\Delta r$, goes from $(r+\Delta r) e^{i(\theta+\Delta \theta)}$ to $r e^{i(\theta+\Delta \theta)}$ along a straight line, and goes from $r e^{i(\theta+\Delta \theta)}$ back to $r e^{i \theta} z$ along the circle $|\zeta|=r$. Let

$$
\Delta r=d\left(\zeta, \zeta^{\prime}\right)
$$

Define $\Delta t=t^{\prime}-t$ and let $\Delta \theta=\Delta t /\left[-2 k\left[(r+\Delta r)^{2 k}-r^{2 k}\right]\right]$. Let $\tilde{\Upsilon}$ be the lift of $\Upsilon$ that has initial height $t$. Using Proposition 4.1.2 we calculate that the final point of $\tilde{\Upsilon}$ has height $-2 k\left[(r+\Delta r)^{2 k}-r^{2 k}\right] \Delta \theta+t=\Delta t+t$. Therefore $\tilde{\Upsilon}$ connects $\zeta$ to $\zeta^{\prime}$. Furthermore, the Euclidean length of $\Upsilon$ is

$$
\begin{aligned}
L(\Upsilon) & =2 \Delta r+(2 r+\Delta r)|\Delta \theta|=2 \Delta r+\frac{(2 r+\Delta r)|\Delta t|}{2 k\left[(r+\Delta r)^{2 k}-r^{2 k}\right]} \\
& \leqslant 2 \Delta r+\frac{(2 r+\Delta r)|\Delta t|}{2 k\left[(\Delta r)^{2 k}+r^{2 k-1} \Delta r\right]} .
\end{aligned}
$$

Using (3.1.3) we see that $|\Delta t| \approx d^{2} r^{2 k-2}+d^{2 k}$ where $d=d\left(\zeta, \zeta^{\prime}\right)$. Therefore, recalling (4.2.15),

$$
\begin{aligned}
L(\Upsilon) & \leq d+\frac{(r+d)\left(d^{2} r^{2 k-2}+d^{2 k}\right)}{d^{2 k}+r^{2 k-1} d} \\
& \approx d+\frac{d^{2} r^{2 k-1}+d^{2 k+1}}{d^{2 k}+r^{2 k-1} d} \\
& =d+d \approx d \quad \text { as required. }
\end{aligned}
$$

4.2.16 LEMMA. If $\zeta$ and $\omega$ are any points in $\partial H_{k}$, then they can be connected by $a$ curve $\tilde{\Upsilon}$ with $\dot{\tilde{\Upsilon}} \in T^{c}$ and $L(\Upsilon)<C d(\zeta, \omega)$.

Proof. First connect $z$ to $w$ with a straight line $\Upsilon_{1}$. Let $\tilde{\Upsilon}_{1}$ be the lift of $\Upsilon_{1}$ and let $\omega^{\prime}=\left(w, s^{\prime}\right)$ be the final point of $\tilde{\Upsilon}_{1}$. By, (3.1.3b), $|z-w|<d(\zeta, \omega)$. Thus by Lemma 4.2.1 $d\left(\zeta, \omega^{\prime}\right) \leqslant L\left(\Upsilon_{1}\right) \leqslant d(\zeta, \omega)$. Hence, by the tringle inequality (3.3.1), $d\left(\omega, \omega^{\prime}\right) \leq d(\zeta, \omega)$. Now by Lemma 4.2 .14 there is a curve $\tilde{\Upsilon}_{2}$ connecting $\omega^{\prime}$ to $\omega$ with $\dot{\tilde{\Upsilon}}_{2} \in T^{c}$ and $L\left(\Upsilon_{2}\right) \leqq d\left(\omega, \omega^{\prime}\right) \leqq d(\zeta, \omega)$. Take $\tilde{\Upsilon}=\tilde{\Upsilon}_{1} \cup \tilde{\Upsilon}_{2}$. 


\subsubsection{Proposition. Define}

$d_{4}(\zeta, \omega)<\delta$ if and only if $\zeta$ and $\omega$ can be connected by a curve whose tangent always lies in the complex tangent space and whose projection in the z plane has length no more than $\delta$.

Then $d_{4}$ is equivalent to the other pseudometrics we have defined.

Proof. This follows from Lemmas 4.2.1 and 4.2.16.

5. Singular integrals. The following sections contain the theorems about singular integral operators that we will apply to the kernel $S$.

5.1 Principal value integrals and $L^{p}$ estimates. The following theorem for $\mathbf{R}^{n}$ appears in Stein [S1]. A version for homogeneous spaces is contained in Coifman and Weiss $[\mathbf{C W}]$, Korányi and Vági $[\mathbf{K o V}]$, and Goodman [Go]. On $\partial H_{k}$ with the pseudometric we have defined we have neither the case of convolution kernels, as in [S1], nor the case of a homogeneous space, as in [CW, KoV, and Go]. However, with various modifications, the proofs in these references work for $\partial H_{k}$. Details of these modifications are given in [Di]. The essential point is that the necessary properties of the pseudometric are a triangle inequality and a volume doubling property, both of which we have established.

Let $K:\left\{\left(\partial H_{k} \times \partial H_{k}\right) \backslash\right.$ diagonal $\} \rightarrow \mathbf{C}$ be measurable. Define $K^{*}(\zeta, \omega)$ to be the complex conjugate of $K(\omega, \zeta), K^{*}(\zeta, \omega)=\overline{K(\omega, \zeta)}$. Theorems 5.1.5 and 5.2.5 say that the following conditions on $K$ and $K^{*}$ suffice for $K$ to define a principal value integral operator that is bounded on $L^{p}\left(\partial H_{k}\right), 1<p<\infty$, and that preserves Lipschitz classes.

(5.1.1) Size condition

$$
|K(\zeta, \omega)| \leqslant B\left(\operatorname{Vol}\left[B_{\zeta}(d(\zeta, \omega))\right]\right)^{-1}, \text { for some } B<\infty .
$$

(5.1.2) Calderón-Zygmund condition

$$
\int_{d(\zeta, \omega)>c d\left(\omega, \omega^{\prime}\right)}\left|K(\zeta, \omega)-K\left(\zeta, \omega^{\prime}\right)\right| d V(\zeta) \leqslant B, \quad \text { for some } c, B<\infty .
$$

$$
\left|K(\zeta, \omega)-K\left(\zeta, \omega^{\prime}\right)\right| \leqslant B \frac{d\left(\omega, \omega^{\prime}\right)}{d(\zeta, \omega) \operatorname{Vol}\left[B_{\zeta}(d(\zeta, \omega))\right]} .
$$

(5.1.3) Mean value zero condition

$$
\int_{R_{1}<d(\zeta, \omega)<R_{2}} K(\zeta, \omega) d V(\omega)=0, \quad \text { for all } 0<R_{1}<R_{2}<\infty .
$$

Observe that, by the integral formula (3.5.1), (5.1.2)' implies (5.1.2).

Define the truncated kernels

$$
K_{\varepsilon}(\zeta, \omega)= \begin{cases}K(\zeta, \omega) & \text { if } d(\zeta, \omega) \geqslant \varepsilon \\ 0 & \text { if } d(\zeta, \omega)<\varepsilon\end{cases}
$$

5.1.5 Theorem. Suppose $K(\zeta, \omega)=\left\{\left(\partial H_{k} \times \partial H_{k}\right) \backslash\right.$ diagonal $\} \rightarrow \mathbf{C}$ and suppose $K$ and $K^{*}$ satisfy (5.1.1), (5.1.2), and (5.1.3). For $f \in L^{p}\left(\partial H_{k}\right), 1<p<\infty$, let

$$
T_{\varepsilon} f(\zeta)=\int K_{\epsilon}(\zeta, \omega) f(\omega) d V(\omega), \quad \varepsilon>0 .
$$


Then

$$
\left\|T_{\varepsilon} f\right\|_{p} \leqslant A_{p}\|f\|_{p}
$$

with $A_{p}$ independent of $f$ and $\varepsilon$. Also, for each $f \in L^{p}, \lim _{\varepsilon \rightarrow 0} T_{\varepsilon} f=T f$ exists in $L^{p}$ norm. The limit operator $T$ also satisfies (5.3.5).

5.2 Preservation of Lipschitz classes.

5.2.1 Definition. For $0<\beta<1$, let

$$
\Gamma_{\beta}=\left\{f \in L^{\infty} \cap C^{0}: \sup _{\zeta, \omega}|f(\zeta)-f(\omega)| / d(\zeta, \omega)^{\beta}<\infty\right\} .
$$

Thus $\Gamma_{\beta}$ is a nonisotropic Lipschitz class defined in terms of the pseudometric of $\S 3$. For $f \in \Gamma_{\beta}$ we let

$$
\|f\|_{\Gamma_{\beta}}=\|f\|_{L^{\infty}}+\sup _{\zeta, \omega} \frac{|f(\zeta)-f(\omega)|}{d(\zeta, \omega)^{\beta}} .
$$

5.2.2 Lemma. Suppose $K$ and $K^{*}$ satisfy (5.1.1), (5.1.2)', and (5.1.3). Define $K_{\varepsilon}, T_{\varepsilon}$, and $T$ as in Theorem 5.1.5. Suppose $0<\beta<1,1<p<\infty$, and $f \in \Gamma_{\beta} \cap L^{p}$. Then

$$
T f(\zeta)=\lim _{\varepsilon \rightarrow 0} \int K_{\varepsilon}(\zeta, \omega) f(\omega) d V(\omega) \text { with convergence pointwise a.e. }
$$

5.2.4 Theorem. Suppose $K$ and $K^{*}$ satisfy (5.1.1), (5.1.2)', and (5.1.3). Define $T$ as in Theorem 5.1.5. Suppose $0<\beta<1,1<p<\infty$. Then there is a constant $A_{p, \beta}<\infty$ such that for $f \in \Gamma_{\beta} \cap L^{p}$

$$
\|T f\|_{\Gamma_{\beta}} \leqslant A_{p, \beta}\left(\|f\|_{\Gamma_{\beta}}+\|f\|_{L^{p}}\right) .
$$

5.2.5 Corollary. Suppose $K$ and $K^{*}$ satisfy (5.1.1), (5.1.2)', and (5.1.3). Define $T$ as in Theorem 5.1.5. Suppose $0<\beta<1, f \in \Gamma_{\beta}$, and $f$ has compact support. Then $T f \in \Gamma_{\beta}$.

6. Estimates for the Szegö kernel. In the next three sections we prove that the Szegö kernel is a singular integral kernel in the sense of $\S 5$. In the fourth section we apply the two main theorems of $\S 5$, Theorems 5.1.5 and 5.2.4, to obtain $L^{p}$ and Lipschitz regularity for the principal value operator defined by the Szegö kernel. In the final section we give the Plemelj formula that relates the projection operator to the principal value operator. Then we deduce, by way of the formula, $L^{p}$ and Lipschitz regularity for the Szegö projection.

6.1 The basic size estimate.

6.1.1 Proposition (Size). $|S(\zeta, \omega)| \approx 1 / \operatorname{Vol}\left[B_{\zeta}(d(\zeta, \omega))\right]$.

Proof. In the notation (3.1.2a)

$$
S=1 / 4 \pi^{2}[P-Q]^{2} P^{k-1} .
$$


Therefore by Lemmas 3.2.1 and 3.2.2, Proposition 3.2.21, and the volume formula in Proposition 3.4.4,

$$
|S| \approx \frac{1}{\delta^{4}\left(|Q|^{k-1}+\delta^{2 k-2}\right)} \approx \frac{1}{\operatorname{Vol}\left[B_{\zeta}(\delta)\right]} \quad \text { where } \delta=d(\zeta, \omega) .
$$

Observe that by the integral formulae 3.5.1 and 3.5.2 the Szegö kernel just misses integrability, both near the diagonal and at infinity. That is,

\subsubsection{Corollary.}

$$
\int_{\omega \in K_{z}}|S(\zeta, \omega)|[d(\zeta, \omega)]^{\alpha} d V(\omega)<\infty \text { and } \int_{\omega \notin K_{z}} \frac{|S(\zeta, \omega)| d V(\omega)}{[d(\zeta, \omega)]^{\alpha}}<\infty
$$

if and only if $\alpha>0$, where $K_{z}$ is any compact neighborhood of $z$.

6.2 Calderon-Zygmund condition. The usual estimate that is made on a singular integral kernel in $\mathbf{R}^{n}$ in order to derive the Calderon-Zygmund condition is $\left|\nabla_{x} K(x, y)\right| \leqslant C|x-y|^{-n-1}$. It is pointed out in Nagel and Stein [NS] that an appropriate generalization to other contexts is

$$
\left|\nabla_{x} K(x, y)\right| \leqslant C\left[|x-y| \cdot \operatorname{Vol} B_{x}(|x-y|)\right]^{-1} .
$$

This is what we use here, with Euclidean distance replaced by the pseudometric $d$ and with $\nabla_{x}$ replaced by the holomorphic tangent vector $Z$.

6.2.1 Lemma. $\left|Z_{\zeta}[S(\zeta, \omega)]\right| \leqslant\left[d(\zeta, \omega) \cdot\left[\operatorname{Vol} B_{\zeta}(d(\zeta, \omega))\right]\right]^{-1}$ where $Z_{\zeta}$ is the holomorphic derivative defined in (2.1.2a).

Proof. We use the notation (3.1.2a) and write $d(\zeta, \omega)=\delta$. Then

$$
\begin{aligned}
Z_{\zeta}[S(\zeta, \omega)] & =\left[\frac{-(k+1) z^{k-1} \bar{z}^{k}}{P^{k}}+\frac{i \bar{w}(s-t)+\bar{w}\left[|w|^{2 k}-|z|^{2 k}\right]}{P^{k}(P-Q)}\right] S(\zeta, \omega) \\
& =[I] S(\zeta, \omega) .
\end{aligned}
$$

Case (i) Suppose $|z|<2 \delta$. Then by (3.2.22b) $|w| \leqslant \delta$; by (3.2.22a) $|s-t| \leq \delta^{2 k}$; by Lemma (3.2.1) $|P| \approx \delta^{2}$; and by Lemma 3.2.2 $|P-Q| \approx \delta^{2}$. Therefore

$$
|I| \leq \frac{\delta^{2 k-1}}{\delta^{2 k}}+\frac{\delta\left(\delta^{2 k}\right)+\delta\left(\delta^{2 k}\right)}{\delta^{2 k} \delta^{2}} \approx \frac{1}{\delta} .
$$

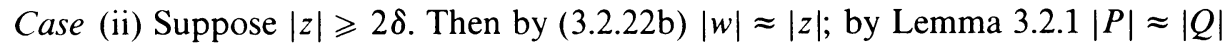
$\approx|z|^{2}$; by (3.1.3) $|s-t| \leq \delta^{2}|z|^{2 k-2}+\left|\operatorname{Im} \bar{z}^{k} w^{k}\right| \leq \delta|z|^{2 k-1}$; and by Lemma 3.2.2 $|P-Q| \approx \delta^{2}$. Therefore

$$
|I| \leq \frac{|z|^{2 k-1}}{|z|^{2 k}}+\frac{|z| \delta|z|^{2 k-1}+|z||z|^{2 k-1} \delta}{|z|^{2 k} \delta^{2}} \approx \frac{1}{\delta} .
$$

Combining $|I| \leq 1 / \delta$ with the estimate in Proposition 6.1.1 on $|S(\zeta, \omega)|$ we obtain the lemma. 


\subsubsection{LemMa. $\bar{Z}_{\zeta} S(\zeta, \omega)=0$.}

Proof. This is a consequence of the holomorphicity of the Szegö kernel in its first variable.

6.2.3 Proposition (CAlderón - Zygmund CONDItion). There exist constants $c$ and $B$ depending only on $k$ with

$$
\int_{\omega \notin B_{\zeta}\left(c d\left(\zeta, \zeta^{\prime}\right)\right)}\left|S(\zeta, \omega)-S\left(\zeta^{\prime}, \omega\right)\right| d V(\omega) \leqslant B .
$$

Proof. Recall that (4.1.1) implies that if $\vec{V}$ lies in the complex part of the tangent space, $T^{c}$, then $\vec{V}=\nu_{1} \operatorname{Re} Z-\nu_{2} \operatorname{Im} Z$, where $\vec{V}=\left(\nu_{1}, \nu_{2}, \nu_{3}\right)$. Since $\bar{Z}_{\zeta}[S(\zeta, \omega)]=0$,

$$
\left(\operatorname{Re} Z_{\zeta}\right)[S(\zeta, \omega)]=\frac{1}{2}\left(Z_{\zeta}+\bar{Z}_{\zeta}\right)[S(\zeta, \omega)]=\frac{1}{2} Z_{\zeta}[S(\zeta, \omega)]
$$

and similarly

$$
\left(\operatorname{Im} Z_{\zeta}\right)[S(\zeta, \omega)]=(1 / 2 i) Z_{\zeta}[S(\zeta, \omega)]
$$

Therefore,

(6.2.4) If $\vec{V} \in T^{c}$ then

$$
\begin{aligned}
\left(\nabla_{\zeta}[S(\zeta, \omega)]\right) \cdot \vec{V} & =\left(\nabla_{\zeta}[S(\zeta, \omega)]\right) \cdot\left(\nu_{1} \operatorname{Re} Z-\nu_{2} \operatorname{Im} Z\right) \\
& =\nu_{1}\left(\operatorname{Re} Z_{\zeta}\right)[S(\zeta, \omega)]-\nu_{2}\left(\operatorname{Im} Z_{\zeta}\right)[S(\zeta, \omega)] \\
& =\frac{1}{2}\left(\nu_{1}+i \nu_{2}\right)\left(Z_{\zeta}[S(\zeta, \omega)]\right),
\end{aligned}
$$

where the $\zeta$ subscript indicates that the differentiation is in $\zeta$, and $\nabla_{\zeta}$ is the ordinary gradient $(\partial / \partial x, \partial / \partial y, \partial / \partial t)$.

Now connect $\zeta^{\prime}$ to $\zeta$ with a curve $\tilde{\Upsilon}:[a, b] \rightarrow \partial H_{k}$ that satisfies $\dot{\tilde{\Upsilon}} \in T^{c}$ and $L(\Upsilon) \leqslant c d\left(\zeta, \zeta^{\prime}\right)$. Such a curve is guaranteed to exist by Lemma 4.2.16. Write $\Upsilon=\left(\gamma_{1}, \gamma_{2}\right)$. Then by $(6.2 .4)$

$$
\left(\nabla_{\zeta}[S(\zeta, \omega)]\right) \cdot \dot{\tilde{\Upsilon}}=\frac{1}{2}\left(\dot{\gamma}_{1}+i \dot{\gamma}_{2}\right)\left(Z_{\zeta}[S(\zeta, \omega)]\right) .
$$

Therefore, using (6.2.5) and Lemma 6.2.1,

$$
\begin{aligned}
\left|S(\zeta, \omega)-S\left(\zeta^{\prime}, \omega\right)\right| & =\left|\int_{a}^{b} \frac{d}{d \tau} S(\tilde{\Upsilon}(\tau), \omega) d \tau\right| \\
& =\left|\int_{a}^{b}\left(\nabla_{\nu}[S(\nu, \omega)]\right)\right|_{\nu=\tilde{\Upsilon}(\tau)} \cdot \dot{\tilde{\Upsilon}}(\tau) d \tau \mid \\
& =\left|\frac{1}{2} \int_{a}^{b}\left(\dot{\gamma}_{1}(\tau)+i \dot{\gamma}_{2}(\tau)\right)\left(Z_{\nu}[S(\nu, \omega)]\right)\right|_{\nu=\tilde{\Upsilon}(\tau)} d \tau \mid \\
& \leqslant \frac{1}{2} \int_{a}^{b}|\dot{\Upsilon}(\tau)|\left|Z_{\nu}[S(\nu, \omega)]\right|_{\nu=\tilde{\Upsilon}(\tau)} \mid d \tau . \\
& \leqslant \frac{1}{2} \int_{a}^{b}|\dot{\Upsilon}(\tau)|\left[d(\tilde{\Upsilon}(\tau), \omega) \cdot \operatorname{Vol} B_{\tilde{\Upsilon}(\tau)}(d(\tilde{\Upsilon}(\tau), \omega))\right]^{-1} d \tau .
\end{aligned}
$$


By Lemma 4.2.1, all of the image of $\tilde{\Upsilon}$ is contained in a ball $B_{\zeta}\left(c_{1} d\left(\zeta, \zeta^{\prime}\right)\right)$, where $c_{1}$ depends only on $k$. Therefore, by the triangle inequality Proposition 3.3.4 and the formula for volume Proposition 3.4.4,

$$
d(\tilde{\Upsilon}(\tau), \omega) \operatorname{Vol} B_{\tilde{\Upsilon}(\tau)}(d(\tilde{\Upsilon}(\tau), \omega)) \approx d(\zeta, \omega) \operatorname{Vol}\left(B_{\zeta} d(\zeta, \omega)\right)
$$

uniformly for all $\tau \in[a, b]$ and all $\omega$ outside some ball $B_{\zeta}\left(c d\left(\zeta, \zeta^{\prime}\right)\right)$. For such $\omega$ (6.2.6) becomes, then,

$$
\begin{aligned}
\left|S(\zeta, \omega)-S\left(\zeta^{\prime}, \omega\right)\right| & \leq\left[d(\zeta, \omega) \operatorname{Vol} B_{\zeta}(d(\zeta, \omega))\right]^{-1} \int_{a}^{b}|\dot{\Upsilon}(\tau)| d \tau \\
& =\left[d(\zeta, \omega) \operatorname{Vol} B_{\zeta}(d(\zeta, \omega))\right]^{-1} L(\Upsilon) \\
& \leq\left[d(\zeta, \omega) \operatorname{Vol} B_{\zeta}(d(\zeta, \omega))\right]^{-1} d\left(\zeta, \zeta^{\prime}\right)
\end{aligned}
$$

Integrating this in $\omega \notin B_{\zeta}\left(c d\left(\zeta, \zeta^{\prime}\right)\right)$ and using the integral formula in Proposition 3.5.1 with $\alpha=1$, we obtain

$$
\begin{aligned}
\int_{\omega \notin B_{\zeta}\left(d d\left(\zeta . \zeta^{\prime}\right)\right)}\left|S(\zeta, \omega)-S\left(\zeta^{\prime}, \omega\right)\right| d V(\omega) & \\
& \leq d\left(\zeta, \zeta^{\prime}\right) \int_{\omega \notin B_{\zeta^{\prime}}\left(c d\left(\zeta . \zeta^{\prime}\right)\right)}\left[d(\zeta, \omega) \operatorname{Vol} B_{\zeta}(d(\zeta, \omega))\right]^{-1} d V(\omega) \\
& \approx d\left(\zeta, \zeta^{\prime}\right) / d\left(\zeta, \zeta^{\prime}\right) \leqslant B \quad \text { for some constant } B . \quad \square
\end{aligned}
$$

6.3 Mean value zero.

6.3.1 Lemma. Let, for $0<a<b, \operatorname{Re} \alpha>1$

$$
J(a, b)=\int_{0}^{\pi} \int_{a}^{b} \sin ^{\alpha-2} \theta \frac{1}{\rho\left(\rho-i e^{i \theta}\right)^{\alpha}} d \rho d \theta .
$$

Then

$$
\lim _{\substack{a \rightarrow 0 \\ b \rightarrow \infty}} J(a, b)=\frac{\pi}{2^{\alpha-1}(\alpha-1)} .
$$

Proof. If $\alpha$ is an integer $\geqslant 2$ the formula (6.3.1a) is Lemma 6.2 of Korányi-Vági [KoV]. We show that (6.3.1a) extends analytically to $\alpha$ with $\operatorname{Re} \alpha>1$ as follows. Clearly $\pi / 2^{\alpha-1}(\alpha-1)$ is analytic in $\alpha$ for $\operatorname{Re} \alpha>1$ and is bounded for $\operatorname{Re} \alpha>t>1$. We show that on the half-planes $\operatorname{Re} \alpha>t>1$, the function $\lim _{a \rightarrow 0 ; h \rightarrow \infty} J(a, b)$ is also analytic in $\alpha$ and that it is bounded by $C_{t}(1+|\alpha|)$. Then, since a function that is analytic in a half-plane and whose growth is at most polynomial is determined by its values at the integers, we obtain the identity (6.3.1a) for all $\alpha$ with $\operatorname{Re} \alpha>1$.

Let

$$
f_{\alpha}(\rho, \theta)=\sin ^{\alpha-2} \theta \frac{1}{\rho\left(\rho-i e^{i \theta}\right)^{\alpha}},
$$

so that

$$
J(a, b)=\int_{0}^{\pi} \int_{a}^{b} f_{\alpha}(\rho, \theta) d \rho d \theta
$$


The obstacle to showing that $\lim _{a \rightarrow 0 ; b \rightarrow \infty} J(a, b)$ is an analytic function of $\alpha$ is the fact that $f_{\alpha}$ is not in $L^{1}([0, \infty) \times[0, \pi])$. We replace $f_{\alpha}$ with a new function that is in $L^{1}$. We do this by subtracting from $f_{\alpha}$ a suitable term $g_{\alpha}$ whose integral on $[a, b] \times[0, \pi]$ is zero for each $a$ and $b$. Observe that the following integral is zero.

$$
\begin{aligned}
\int_{0}^{\pi} \int_{a}^{b} \sin ^{\alpha-2} \theta \frac{1}{\rho\left(-i e^{i \theta}\right)^{\alpha}} d \rho d \theta & =-\left(\frac{1}{2}\right)^{\alpha-2}\left[\int_{a}^{b} \frac{d \rho}{\rho}\right] \int_{0}^{\pi}\left(e^{i \theta}-e^{-i \theta}\right)^{\alpha-2} e^{-i \alpha \theta} d \theta \\
& =-\left(-\frac{1}{2}\right)^{\alpha-2}\left[\int_{a}^{b} \frac{d \rho}{\rho}\right] \int_{0}^{\pi}\left(1-e^{-2 i \theta}\right)^{\alpha-2} e^{-2 i \theta} d \theta \\
& =-\left.\left(-\frac{1}{2}\right)^{\alpha-2}\left[\int_{a}^{b} \frac{d \rho}{\rho}\right] \frac{\left(1-e^{-2 i \theta}\right)^{\alpha-1}}{(\alpha-1)(2 i)}\right|_{0} ^{\pi}=0 .
\end{aligned}
$$

Therefore, let

$$
g_{\alpha}(\rho, \theta)=\left(\sin ^{\alpha-2} \theta\right) \frac{1}{\rho\left(-i e^{i \theta}\right)^{\alpha}} \chi_{[0,1]}(\rho) .
$$

Then,

$$
\begin{aligned}
J(a, b) & =\int_{0}^{\pi} \int_{a}^{1}\left[f_{\alpha}(\rho, \theta)-g_{\alpha}(\rho, \theta)\right] d \rho d \theta+\int_{0}^{\pi} \int_{1}^{b} f_{\alpha}(\rho, \theta) d \rho d \theta \\
& =\int_{0}^{\pi} \int_{a}^{b}\left[f_{\alpha}(\rho, \theta)-g_{\alpha}(\rho, \theta)\right] d \rho d \theta .
\end{aligned}
$$

Now we verify that $f_{\alpha}-g_{\alpha}$ is integrable. First note that for $(\rho, \theta) \in[0, \infty) \times[0, \pi]$, $\left|\rho-i e^{i \theta}\right| \geqslant 1$. Therefore, it suffices to examine $f_{\alpha}-g_{\alpha}$ for $\rho$ near $0, \rho$ near $\infty, \theta$ near 0 , and $\theta$ near $\pi$. For small $\rho$,

$$
\begin{aligned}
\left|\frac{1}{\rho}\left(\frac{1}{\left(\rho-i e^{i \theta}\right)^{\alpha}}-\frac{1}{\left(-i e^{i \theta}\right)^{\alpha}}\right)\right| & =\frac{1}{\rho}\left|\left(\frac{\rho}{-i e^{i \theta}}+1\right)^{-\alpha}-1\right| \\
& \approx \frac{1}{\rho}\left|1+\frac{\alpha \rho}{i e^{i \theta}}-1\right|=\frac{1}{\rho}|\alpha \rho|=|\alpha| .
\end{aligned}
$$

For large $\rho$,

$$
\left|\frac{1}{\rho} \frac{1}{\left(\rho-i e^{i \theta}\right)^{\alpha}}\right| \approx\left|\frac{1}{\rho^{1+\alpha}}\right|=\frac{1}{\rho^{1+\operatorname{Re} \alpha}} .
$$

For small $\theta,\left|\sin ^{\alpha-2} \theta\right| \approx|\theta|^{\operatorname{Re} \alpha-2}$, and for $\theta$ near $\pi,\left|\sin ^{\alpha-2} \theta\right| \approx|\pi-\theta|^{\operatorname{Re} \alpha-2}$. Hence,

$$
f_{\alpha}(\rho, \theta)-g_{\alpha}(\rho, \theta) \in L^{1}([0, \infty) \times[0, \pi]) \quad \text { if } \operatorname{Re} \alpha>1 .
$$

Moreover,

for each compact subset $K$ of $\{\operatorname{Re} \alpha>1\}$ there is a $C_{K}$ with $\left\|f_{\alpha}-g_{\alpha}\right\|_{L^{1}([0, \infty) \times[0, \pi])} \leqslant C_{K}$ for all $\alpha \in K$, and for each $t>1$ there is a $C_{t}$ with $\left\|f_{\alpha}-g_{\alpha}\right\|_{L^{1}([0, \infty) \times[0, \pi])} \leqslant$ $C_{t}(|\alpha|+1)$ for all $\alpha$ with $\operatorname{Re} \alpha>t$.

Now we can easily show that $\lim _{a \rightarrow 0 ; h \rightarrow \infty} J(a, b)$ is an analytic function of $\alpha$ for $\operatorname{Re} \alpha>1$. Consider the family $\{J(a, b)\}_{a, b \in R}$ of functions analytic in $\alpha$. By (6.3.2b), 
for each compact subset $K$ of $\{\operatorname{Re} \alpha>1\}$,

$$
\begin{aligned}
|J(a, b)| & =\left|\int_{0}^{\pi} \int_{a}^{b}\left(f_{\alpha}-g_{\alpha}\right) d \rho d \theta\right| \leqslant \int_{0}^{\pi} \int_{0}^{\infty}\left|f_{\alpha}-g_{\alpha}\right| d \rho d \theta \\
& =\left\|f_{\alpha}-g_{\alpha}\right\|_{L^{1}} \leqslant C_{K}
\end{aligned}
$$

for all $\alpha \in K$. That is, the family $\{J(a, b)\}_{a, b \in R}$ is uniformly bounded on compact subsets of $\{\operatorname{Re} \alpha>1\}$. Therefore, by Montel's theorem, there is an analytic function $F(\alpha)$ and a subsequence $\left\{J\left(a_{i}, b_{i}\right)\right\}$ with $a_{i} \rightarrow 0$ and $b_{i} \rightarrow \infty$ such that $\lim _{i \rightarrow \infty} J\left(a_{i}, b_{i}\right)=F(\alpha)$. However, by (6.3.2a) and the Lebesgue dominated convergence theorem,

$$
\lim _{\substack{a \rightarrow 0 \\ b \rightarrow \infty}} J(a, b)=\lim _{\substack{a \rightarrow 0 \\ b \rightarrow \infty}} \int_{0}^{\pi} \int_{a}^{b}\left(f_{\alpha}-g_{\alpha}\right) d \rho d \theta=\int_{0}^{\pi} \int_{0}^{\infty}\left(f_{\alpha}-g_{\alpha}\right) d \rho d \theta
$$

exists for each $\alpha$. Hence

$$
\lim _{a \rightarrow 0: b \rightarrow \infty} J(a, b)=F(\alpha) .
$$

In particular, $\lim _{a \rightarrow 0 ; h \rightarrow \infty} J(a, b)$ is an analytic function of $\alpha$ for $\operatorname{Re} \alpha>1$. Also, by (6.3.2c), for each $t>1, \quad \lim _{a \rightarrow 0 ; h \rightarrow \infty} J(a, b) \mid \leqslant C_{t}(|\alpha|+t)$ for $\operatorname{Re} \alpha>1$. The lemma now follows by the argument outlined at the beginning of the proof.

\subsubsection{Proposition.}

$$
\lim _{\varepsilon \rightarrow 0} \lim _{\mu \rightarrow 0} \int_{B_{\xi_{j}}^{d_{3}}(\varepsilon)} S\left(\zeta_{\mu}, \omega\right) d V(\omega)=\frac{1}{2},
$$

where $\zeta_{\mu}=\left(z, t+i|z|^{2 k}+i \mu\right)$. Here $d_{3}$ is the pseudometric of (3.2.24).

Proof. First we take the case in which $\zeta_{0}$ is a weak point. Then we treat the case in which $\zeta_{0}$ is a strong point by reducing the calculation to the corresponding calculation on the Heisenberg group, a calculation done in Korányi and Vági [KoV]. In both cases the final computation is given by the formula in Lemma 6.3.1.

Case (i). Assume $z=0$. Then

$$
\begin{aligned}
B_{\zeta_{0}}^{d_{3}}(\varepsilon) & =\left\{(w, s):\left|\frac{i}{2}(s-t)+\frac{|w|^{2 k}}{2}\right|<\frac{\varepsilon^{2 k}}{2} ;|w|<\varepsilon\right\} \\
& =\left\{(w, s):\left|\frac{i}{2}(s-t)+\frac{|w|^{2 k}}{2}\right|<\frac{\varepsilon^{2 k}}{2}\right\} .
\end{aligned}
$$

Therefore, the limit (6.3.5) is

(6.3.6)

$$
\begin{aligned}
\lim _{\varepsilon \rightarrow 0} \lim _{\mu \rightarrow 0} \frac{1}{4 \pi^{2}} & \iiint_{\left.|(i / 2)[s-t]+| w\right|^{2 k} / 2 \mid<\varepsilon^{2 k} / 2} \frac{d(\operatorname{Re} w) d(\operatorname{Im} w) d s}{\left((i / 2)[s-t]+\mu / 2+|w|^{2 k} / 2\right)^{(k+1) / k}} \\
=\lim _{\varepsilon \rightarrow 0} \lim _{\mu \rightarrow 0} \lim _{h \rightarrow 0} \frac{1}{4 \pi^{2}} & \iiint_{h / 2<\left.|(i / 2)[s-t]+| w\right|^{2 k} / 2 \mid<\varepsilon^{2 k} / 2} \\
& \times \frac{d(\operatorname{Re} w) d(\operatorname{Im} w) d s}{\left((i / 2)[s-t]+\mu / 2+|w|^{2 k} / 2\right)^{(k+1) / k}} .
\end{aligned}
$$


We factor the one half from the denominator, use the dilation $s-t=\mu\left(s^{\prime}-t^{\prime}\right)$, $w=\mu^{1 / 2 k} w^{\prime}$, and then drop the primes. The integral on the second line of (6.3.6) becomes

$$
\frac{2^{(1-k) / k}}{\pi^{2}} \iiint_{h / \mu<\left.|i[s-t]+| w\right|^{2 k} \mid<e^{2 k} / \mu} \frac{d(\operatorname{Re} w) d(\operatorname{Im} w) d s}{\left(i(s-t)+1+|w|^{2 k}\right)^{(k+1) / k}} .
$$

Using polar coordinates $(r, \theta)$ for $w$ and performing the trivial $\theta$ integration, we obtain

$$
\frac{2^{1 / k}}{\pi} \underset{\substack{h / \mu<\left|(i / 2)[s-t]+r^{2 k}\right|<\varepsilon^{2 k} / \mu \\ r \geqslant 0}}{\iint^{\left(i(s-t)+1+r^{2 k}\right)^{(k+1) / k}} .}
$$

The change of variables $s-t=-(1 / \rho) \cos \theta, r^{2 k}=(1 / \rho) \sin \theta$ converts the integral to

$$
\frac{2^{(1-k) / k}}{\pi k} \int_{\substack{\mu / \varepsilon^{2 k}<\rho<\mu / h \\ 0 \leqslant \theta \leqslant \pi}} \frac{(\sin \theta)^{(1-k) / k}}{\rho\left(\rho-i e^{i \theta}\right)^{(k+1) / k}} d \rho d \theta .
$$

Now taking $\lim _{\varepsilon \rightarrow 0} \lim _{\mu \rightarrow 0} \lim _{h \rightarrow 0}$ we have that (6.3.6) becomes

$$
\left(\frac{2^{(1-k) / k}}{\pi k}\right) \lim _{\substack{a \rightarrow 0 \\ b \rightarrow \infty}} \int_{0}^{\pi} \int_{a}^{b} \frac{(\sin \theta)^{(1-k) / k}}{\rho\left(\rho-i e^{i \theta}\right)^{(k+1) / k}} d \rho d \theta .
$$

Finally, (6.3.7) may be found from Lemma 6.3 .1 by taking $\alpha=(k+1) / k$. We obtain that (6.3.7) is $\frac{1}{2}$.

Case (ii). Assume $z \neq 0$. The limit on the left side in (6.3.5) is then

$$
\begin{array}{r}
\lim _{\varepsilon \rightarrow 0} \lim _{\mu \rightarrow 0} \frac{1}{4 \pi^{2}} \iiint_{B_{S_{0}}^{d_{3}}(\varepsilon)} \frac{d(\operatorname{Re} w) d(\operatorname{Im} w) d s}{\left[\left((i / 2)[s-t]+\left(|z|^{2 k}+|w|^{2 k}\right) / 2+\mu / 2\right)^{1 / k}-z \bar{w}\right]^{2}} \\
\quad \times\left[(i / 2)[s-t]+\left(|z|^{2 k}+|w|^{2 k}\right) / 2+\mu / 2\right]^{(k-1) / k} .
\end{array}
$$

We show that in the limit both the integrand and the region of integration are essentially those that arise on the Heisenberg group. We adopt the following notation, which is similar to (3.1.2a) used in Chapter 3.

$$
\begin{gathered}
P_{\mu}=\left(\frac{i}{2}[s-t]+\frac{|z|^{2 k}+|w|^{2 k}}{2}+\frac{\mu}{2}\right)^{1 / k}, \quad Q=z \bar{w} . \\
P_{\mu}^{*}=\left(|w|^{2 k}+\frac{\mu}{2}\right)^{1 / k}, \quad Q^{*}=|w|^{2} .
\end{gathered}
$$

Here, $P_{\mu}^{*}$ and $Q^{*}$ are obtained from $P_{\mu}$ and $Q$ by setting $\omega=\zeta$. In this notation (6.3.8) is

$$
\lim _{\varepsilon \rightarrow 0} \lim _{\mu \rightarrow 0} \frac{1}{4 \pi^{2}} \iiint_{B_{\xi_{0}}^{\lambda_{2}}(\varepsilon)} \frac{d(\operatorname{Re} w) d(\operatorname{Im} w) d s}{\left[P_{\mu}-Q\right]^{2} P_{\mu}^{k-1}} .
$$

We now modify the integrand. 
Note that $\lim _{\omega \rightarrow \zeta} P_{\mu}=P_{\mu}^{*}$ and that, for $z \neq 0, P_{\mu}$ and $P_{\mu}^{*}$ are never zero for $\zeta$, $\omega \in \bar{H}^{k}$. Therefore,

$$
\frac{1}{\left[P_{\mu}-Q\right]^{2}}\left[\frac{1}{\left(P_{\mu}^{*}\right)^{k-1}}-\frac{1}{P_{\mu}^{k-1}}\right]
$$

is locally integrable (uniformly in $\mu$ ). Hence

$$
\lim _{\varepsilon \rightarrow 0} \lim _{\mu \rightarrow 0} \frac{1}{4 \pi^{2}} \iiint_{B_{S_{3}}^{d_{3}}(\varepsilon)} \frac{1}{\left[P_{\mu}-Q\right]^{2}}\left[\frac{1}{\left(P_{\mu}^{*}\right)^{k-1}}-\frac{1}{P_{\mu}^{k-1}}\right] d(\operatorname{Re} w) d(\operatorname{Im} w) d s=0 .
$$

Adding this term to (6.3.10) and noting that $\lim _{\mu \rightarrow 0} P_{\mu}^{*}=|w|^{2},(6.3 .10)$ becomes

$$
\text { (6.3.11) } \begin{aligned}
\lim _{\varepsilon \rightarrow 0} \lim _{\mu \rightarrow 0} \frac{1}{4 \pi^{2}} \iiint_{B_{s_{0}}^{J_{3}}(\varepsilon)} \frac{1}{\left(P_{\mu}^{*}\right)^{k-1}} \frac{d(\operatorname{Re} w) d(\operatorname{Im} w) d s}{\left[P_{\mu}-Q\right]^{2}} \\
=\frac{1}{4 \pi^{2}} \lim _{\varepsilon \rightarrow 0} \lim _{\mu \rightarrow 0} \iiint_{B_{s_{0}}^{d_{3}}(\varepsilon)} \frac{1}{|w|^{2 k-2}} \frac{d(\operatorname{Re} w) d(\operatorname{Im} w) d s}{\left[P_{\mu}-Q\right]^{2}} .
\end{aligned}
$$

Next we convert $1 /\left[P_{\mu}-Q\right]^{2}$ to $1 /\left[P_{\mu}^{k}-Q^{k}\right]^{2}$. Let

$$
\pi_{\mu}=\prod_{j=1}^{k-1}\left(P_{\mu}-Q e^{2 \pi i j / k}\right)^{2} \text { and } \pi_{\mu}^{*}=\prod_{j=1}^{k-1}\left(P_{\mu}^{*}-Q e^{2 \pi i j / k}\right)^{2} .
$$

Then

$$
\frac{1}{\left[P_{\mu}-Q\right]^{2}}=\frac{1}{\left[P_{\mu}-Q\right]^{2}} \frac{\pi_{\mu}^{*}}{\pi_{\mu}}+\frac{1}{\left[P_{\mu}-Q\right]^{2}} \frac{\pi_{\mu}-\pi_{\mu}^{*}}{\pi_{\mu}} .
$$

Now, $\lim _{\omega \rightarrow \zeta} \pi_{\mu}=\pi_{\mu}^{*}$ and, for $z \neq 0, \pi_{\mu}$ is never zero. Therefore the second term on the right in (6.3.12) is locally integrable. Herce its integral on $B_{\zeta_{0}}^{d_{3}}(\varepsilon)$ vanishes as $\varepsilon \rightarrow 0$ and the term on the second line of (6.3.11) becomes

$$
\frac{1}{4 \pi^{2}} \lim _{\varepsilon \rightarrow 0} \lim _{\mu \rightarrow 0} \iint_{B_{s_{0}}^{U_{3}}(\varepsilon)} \pi_{\mu}^{*} \frac{1}{|w|^{2 k-2}} \frac{d(\operatorname{Re} w) d(\operatorname{Im} w) d s}{\left[P_{\mu}-Q\right]^{2} \pi_{\mu}} .
$$

Note that

$$
\left[P_{\mu}-Q\right]^{2} \pi_{\mu}=\prod_{j=0}^{k-1}\left(P_{\mu}-Q e^{2 \pi i j / k}\right)^{2}=\left(P_{\mu}^{k}-Q^{k}\right)^{2} .
$$

Also, in the limit $\pi_{\mu}^{*}$ becomes

$$
\prod_{j=1}^{k-1}\left(|w|^{2}-|w|^{2} e^{2 \pi i j / k}\right)^{2}=\left(|w|^{2}\right)^{2 k-2} \prod_{j=1}^{k-1}\left(1-e^{2 \pi i j / k}\right)^{2}=k^{2}\left(|w|^{2}\right)^{2 k-2} .
$$

Thus (6.3.13) becomes

$$
\frac{k^{2}}{4 \pi^{2}} \lim _{\varepsilon \rightarrow 0} \lim _{\mu \rightarrow 0} \iiint_{B_{s_{n}^{2}}^{J_{1}}(\varepsilon)}|w|^{2 k-2} \frac{d(\operatorname{Re} w) d(\operatorname{Im} w) d s}{\left[P_{\mu}^{k}-Q^{k}\right]^{2}} .
$$


The integrand of (6.3.14) transforms to the Szegö kernel times the usual volume form $d(\operatorname{Re} \nu) d(\operatorname{Im} \nu) d s$ on the Heisenberg group under the straightforward change of variables

$$
\nu=w^{k} \quad(s \text { unchanged }) .
$$

Before making the change, however, we make some observations about the region of integration, $B_{\zeta_{0}}^{d_{3}}(\varepsilon)$.

(6.3.16) Fix $\zeta_{0}=(z, t)$. For $\varepsilon<(\sin (\pi / k))|z|$, the set

$$
\begin{aligned}
B^{\prime}=\left\{(w, s):\left|\frac{i}{2}(s-t)+\frac{|z|^{2 k}+|w|^{2 k}}{2}-z^{k} \bar{w}^{k}\right|\right. \\
\left.\quad<\frac{(\sin (\pi / k))^{2 k-2}}{4}|z|^{2 k-2} \varepsilon^{2}+\frac{\varepsilon^{2 k}}{2}\right\}
\end{aligned}
$$

is a disjoint union of $k$ sets $B_{j}$. One $B_{j}$ lies in each of the cylinders

$$
C_{j}=\left\{(w, s):\left|w-z e^{2 \pi i j / k}\right|<\varepsilon\right\}, \quad j=0, \ldots, k-1 .
$$

Also, each $B_{j}$ is the rotation in the $z$ plane by $e^{2 \pi i j / k}$ of the ball

$$
\begin{aligned}
B_{\zeta_{0}}^{d_{3}}(\varepsilon)=\left\{(w, s): \mid \frac{i}{2}(s-t)\right. & +\frac{|z|^{2 k}+|w|^{2 k}}{2}-z^{k} \bar{w}^{k} \mid \\
& \left.<\frac{(\sin (\pi / k))^{2 k-2}}{4}|z|^{2 k-2} \varepsilon^{2}+\frac{\varepsilon^{2 k}}{2} ;|w-z|<\varepsilon\right\} .
\end{aligned}
$$

To verify these claims, note that for all $j \in\{0,1, \ldots, k-1\}$ except possibly for one such $j_{0},\left|w-z e^{2 \pi i j / k}\right| \geqslant(\sin (\pi / k))|z|$. On the other hand, if $\omega \in B^{\prime}$ then for $\varepsilon<(\sin (\pi / k))|z|$,

$$
\begin{aligned}
\left|w^{k}-z^{k}\right|^{2} & =2 \operatorname{Re}\left[\frac{i}{2}(s-t)+\frac{|z|^{2 k}+|w|^{2 k}}{2}-z^{k} \bar{w}^{k}\right] \\
& <\frac{(\sin (\pi / k))^{2 k-2}}{2}|z|^{2 k-2} \varepsilon^{2}+\varepsilon^{2 k} \\
& <(\sin (\pi / k))^{2 k-2}|z|^{2 k-2} \varepsilon^{2} .
\end{aligned}
$$

Hence

$$
\left|w-z e^{2 \pi i j_{0} / k}\right|^{2}=\frac{\left|w^{k}-z^{k}\right|^{2}}{\prod_{j=0 ; j \neq j_{0}}^{k-1}\left|w-z e^{2 \pi i j / k}\right|^{2}} \leqslant \frac{(\sin (\pi / k))^{2 k-2}|z|^{2 k-2} \varepsilon^{2}}{(\sin (\pi / k))^{2 k-2}|z|^{2 k-2}}=\varepsilon^{2} .
$$

Therefore each $\omega \in B^{\prime}$ lies in at least one of the cylinders $C_{j}$. On the other hand, for $\varepsilon<(\sin (\pi / k))|z|$ the $C_{j}$ are disjoint. Thus each $\omega \in B^{\prime}$ lies in exactly one of the $C_{j}$. The remaining claim, the symmetry, is clear.

Returning to the integral (6.3.14), we observe that the integrand is unchanged under $w \rightarrow w e^{2 \pi i j / k}$. Therefore, by (6.3.16), we see that if we drop the condition $|z-w|<\varepsilon$ in the definition of $B_{\zeta_{0}}^{d_{3}}(\varepsilon)$, then we obtain $k$ copies of the original integral. Thus, dropping the condition and dividing by $k$ to compensate, (6.3.14) 
becomes

$(6.3 .17)$

$$
\begin{aligned}
& \frac{k}{4 \pi^{2}} \lim _{\varepsilon \rightarrow 0} \lim _{\mu \rightarrow 0} \iiint_{\left|(i / 2)(s-t)+\left(|z|^{2 k}+|w|^{2 k}\right) / 2-z^{k} \bar{w}^{k}\right|<\left[(\sin (\pi / k))^{2 k-2} / 4\right]|z|^{2 k-2} \varepsilon^{2}+\varepsilon^{2 k} / 2} \\
& \times \frac{|w|^{2 k-2} d(\operatorname{Re} w) d(\operatorname{Im} w) d s}{\left[P_{\mu}^{k}-Q^{k}\right]^{2}} \\
& =\frac{k}{4 \pi^{2}} \lim _{\varepsilon \rightarrow 0} \lim _{\mu \rightarrow 0} \iiint_{\left|(i / 2)(s-t)+\left(|z|^{2 k}+|w|^{2 k}\right) / 2-z^{k} \bar{w}^{k}\right|<\left[(\sin (\pi / k))^{2 k-2} / 4\right]|z|^{2 k-2} \varepsilon^{2}+\varepsilon^{2 k} / 2} \\
& \times \frac{|w|^{2 k-2} d(\operatorname{Re} w) d(\operatorname{Im} w) d s}{\left[\frac{i}{2}(s-t)+\frac{|z|^{2 k}+|w|^{2 k}}{2}+\frac{\mu}{2}-z^{k} \bar{w}^{k}\right]^{2}} .
\end{aligned}
$$

Now we make the change of variables $v=w^{k}$ and also write $u=z^{k}$. Then, since the map $w \rightarrow v$ is $k$ to one, we multiply by $k$. Now (6.3.17) becomes

$$
\begin{aligned}
\frac{k^{2}}{4 \pi^{2}} \lim _{\varepsilon^{\prime} \rightarrow 0} \lim _{\mu \rightarrow 0} & \iiint_{\left|(i / 2)[s-t]+\left(|u|^{2}+|v|^{2}\right) / 2-u \bar{v}\right|<\varepsilon^{\prime}} \\
& \times \frac{1}{k^{2}} \frac{d(\operatorname{Re} v) d(\operatorname{Im} v) d s}{\left[(i / 2)(s-t)+\left(|u|^{2}+|v|^{2}\right) / 2+\mu / 2-u \bar{v}\right]^{2}} .
\end{aligned}
$$

Finally, the $k^{2}$ 's cancel and (6.3.18) is the Heisenberg group calculation. By the corollary to Lemma 6.2 in Korányi and Vági [ KoV], (6.3.18) is $\frac{1}{2}$.

6.3.19 Corollary. For $\varepsilon>0$, define

$$
I_{\varepsilon}=\lim _{\mu \rightarrow 0} \int_{B_{\zeta_{0}}^{d_{3}(\varepsilon)}} S\left(\zeta_{\mu}, \omega\right) d V(\omega), \quad \text { where } \zeta_{\mu}=\left(z, t+i\left(|z|^{2 k}+\mu\right)\right) .
$$

Then, for all $\varepsilon>0, I_{\varepsilon}=\frac{1}{2}$.

Proof. Using the dilations $w=\mu^{1 / 2 k} w^{\prime}, z=\mu^{1 / 2 k} z^{\prime}, s-t=\mu\left(s^{\prime}-t^{\prime}\right)$, and recalling the homogeneity properties (see (3.3.6)) of the pseudometric and the kernel,

$$
I_{\varepsilon}=\lim _{\mu \rightarrow 0} \int_{B_{\zeta_{0}^{\prime}}^{d_{3}\left(\varepsilon / \mu^{1 / 2 k}\right)}} S\left(\zeta_{1}^{\prime}, \omega^{\prime}\right) d V\left(\omega^{\prime}\right)=\lim _{R \rightarrow \infty} \int_{B_{\zeta_{0}^{\prime}}^{d_{3}(R)}} S\left(\zeta_{1}^{\prime}, \omega^{\prime}\right) d V\left(\omega^{\prime}\right) .
$$

Therefore $I_{\varepsilon}$ does not depend on $\varepsilon$. On the other hand, by Proposition 6.3.4, $\lim _{\varepsilon \rightarrow 0} I_{\varepsilon}=\frac{1}{2}$. Hence, $I_{\varepsilon} \equiv \frac{1}{2}$.

\subsubsection{Proposition (Mean value zero).}

$$
\int_{a<d_{3}\left(\zeta_{0}, \omega\right)<b} S\left(\zeta_{0}, \omega\right) d V(\omega)=0 \quad \text { for all } 0<a \leqslant b
$$

Here $d_{3}$ is the pseudometric defined in (3.2.24). 
Proof. Let $\zeta_{\mu}=\left(z, t+i\left(|z|^{2 k}+\mu\right)\right)$. Then, using the notation and result of Corollary 6.3.19,

$$
\begin{aligned}
\int_{a<d_{3}\left(\zeta_{0}, \omega\right)<b} S\left(\zeta_{0}, \omega\right) d V(\omega) & =\lim _{\mu \rightarrow 0} \int_{a<d_{3}\left(\zeta_{0}, \omega\right)<b} S\left(\zeta_{\mu}, \omega\right) d V(\omega) \\
& =I_{b}-I_{a}=0 . \quad \square
\end{aligned}
$$

6.4. $L^{p}$ bounds and preservation of Lipschitz classes. We now have enough information about the kernel $S$ to apply Theorems 5.1.5 and 5.2.4.

REMARK. Note that for our operator we have an alternative to the usual methods for obtaining the $L^{2}$ parts of Theorem 5.1.5. The special operator PVS clearly inherits boundedness on $L^{2}\left(\partial H_{k}\right)$ directly from the Szegö projection, a projection in $L^{2}\left(\partial H_{k}\right)$, through the Plemelj formula given below in Theorem 6.5.1. From this it is possible to deduce $L^{2}$ bounds for the truncated operators as well, giving the $L^{2}$ information required in the proof of Theorem 5.1.5. However, in order to complete the proof of $L^{p}$ boundedness we still require all three estimates (5.1.1), (5.1.2), and (5.1.3) on the kernel. The first two are needed to obtain that the truncated operators are weak type $(1,1)$ and the third is needed to pass from the truncated operators to the principal value operator.

6.4.1 TheOREM ( $L^{p}$ Bounds). For $f \in L^{p}\left(\partial H_{k}\right), 1<p<\infty$, and for $\varepsilon>0$, let

$$
S_{\varepsilon} f(\zeta)=\int_{d_{3}(\zeta, \omega)>\varepsilon} f(\omega) S(\zeta, \omega) d V(\omega) .
$$

Then there is an $A_{p}<\infty$ depending only on $k$ and $p$ such that

$$
\left\|S_{\varepsilon} f\right\|_{p} \leqslant A_{p}\|f\|_{p} .
$$

Also, $\lim _{\varepsilon \rightarrow 0} S_{\varepsilon} f=\operatorname{PVS} f$ exists in $L^{p}$ norm and

$$
\|\operatorname{PVS} f\|_{p} \leqslant A_{p}\|f\|_{p} .
$$

Proof. Propositions 6.1.1, 6.2.3, and 6.3.20, with the observation that $S^{*}(\zeta, \omega)$ $=\overline{S(\omega, \zeta)}=S(\zeta, \omega)$, imply that $S$ satisfies the hypotheses of Theorem 5.1.5.

6.4.2 Theorem (Preservation of LipsChitz Classes). Suppose $0<\beta<1,1<p$ $<\infty$, and $f \in \Gamma_{\beta} \cap L^{p}$. Let $g=\operatorname{PVS} f$, where $\operatorname{PVS} f$ is defined in the statement of Theorem 6.4.1. Then $\|g\|_{\Gamma_{\beta}} \leqslant A_{p, \beta}\left(\|f\|_{\Gamma_{\beta}}+\|f\|_{L^{p}}\right)$, where $A_{p, \beta}$ depends only on $k, \beta$, and $p$.

Proof. Proposition 6.1.1, inequality (6.2.7), Proposition 6.3.20, and the fact $S^{*}=S$, imply that $S$ satisfies the hypotheses of Theorem 5.2.4.

6.5 The Plemelj jump formula and the Szegö projection. Recall that, for $f \in L^{2}\left(\partial H_{k}\right)$, the Szegö projection is

$$
\mathbf{S} f(\zeta)=\lim _{\mu \rightarrow 0} \int f(\omega) S\left(\zeta_{\mu}, \omega\right) d V(\omega),
$$

where $\zeta_{\mu}=\left(z, t+i\left(|z|^{2 k}+\mu\right)\right)$ and the limit is taken in $L^{2}$. 
6.5.1 Theorem (Plemelu Jump formula). Suppose $0<\beta<1, f \in \Gamma_{\beta}$, and $f$ has compact support. Then $\mathbf{S} f(\zeta)=\frac{1}{2} f(\zeta)+\operatorname{PVS} f(\zeta)$ for almost every $\zeta \in \partial H_{k}$.

Proof. The conditions $f \in \Gamma_{\beta}$ and $\operatorname{supp} f$ is compact imply $f \in L^{2}$. Hence, the $L^{2}$ limit

$$
\lim _{\mu \rightarrow 0} \int f(\omega) S\left(\zeta_{\mu}, \omega\right) d V(\omega)=\mathbf{S} f(\zeta)
$$

exists. On the other hand, for each $\zeta \in \partial H_{k}$ and $\varepsilon>0$,

$$
\begin{aligned}
\int f(\omega) S\left(\zeta_{\mu}, \omega\right) d V(\omega)= & \int_{d_{3}(\zeta, \omega)>\varepsilon} f(\omega) S\left(\zeta_{\mu}, \omega\right) d V(\omega) \\
& +\int_{d_{3}(\zeta, \omega)<\varepsilon} f(\omega) S\left(\zeta_{\mu}, \omega\right) d V(\omega) \\
= & \int_{d_{3}(\zeta, \omega)>\varepsilon} f(\omega) S\left(\zeta_{\mu}, \omega\right) d V(\omega) \\
& +\int_{d_{3}(\zeta, \omega)<\varepsilon}[f(\omega)-f(\zeta)] S\left(\zeta_{\mu}, \omega\right) d V(\omega) \\
& +f(\zeta) \int_{d_{3}(\zeta, \omega)<\varepsilon} S\left(\zeta_{\mu}, \omega\right) d V(\omega) .
\end{aligned}
$$

Let $\mu \rightarrow 0$. Since $f(\omega) S\left(\zeta_{\mu}, \omega\right)$ is continuous for $d_{3}(\zeta, \omega)>\varepsilon$, the first term on the last line of (6.5.2) becomes $\int_{d_{3}(\zeta, \omega)>\varepsilon} f(\omega) S(\zeta, \omega) d V(\omega)$. Similarly, the second integrand is locally integrable and the second term becomes

$$
\int_{d_{3}(\zeta, \omega)<\varepsilon}[f(\omega)-f(\zeta)] S(\zeta, \omega) d V(\omega) .
$$

By Proposition 6.3.4 the third term becomes $\frac{1}{2} f(\zeta)$. Hence, for each $\zeta \in \partial H_{k}$ and $\varepsilon>0$,

$$
\begin{aligned}
\lim _{\mu \rightarrow 0} \int f(\omega) S\left(\zeta_{\mu}, \omega\right) d V(\omega) & =\int_{d_{3}(\zeta, \omega)>\varepsilon} f(\omega) S(\zeta, \omega) d V(\omega) \\
& +\int_{d_{3}(\zeta, \omega)<\varepsilon}[f(\omega)-f(\zeta)] S(\zeta, \omega) d V(\omega)+\frac{1}{2} f(\zeta) .
\end{aligned}
$$

Letting $\varepsilon \rightarrow 0$ on the right in (6.5.3), the first term becomes PVS $f(\zeta)$. Since the integrand in the second term is integrable, the second term tends to zero as $\varepsilon \rightarrow 0$. The third term is unchanged. Hence, for each $\zeta \in \partial H_{k}$

$$
\lim _{\mu \rightarrow 0} \int f(\omega) S\left(\zeta_{\mu}, \omega\right) d V(\omega)=\operatorname{PVS} f(\zeta)+\frac{1}{2} f(\zeta)
$$

Since the $L^{2}$ limit of the left side is $\mathbf{S} f$ we obtain

$$
\mathbf{S} f(\zeta)=\operatorname{PVS} f(\zeta)+\frac{1}{2} f(\zeta) \quad \text { almost everywhere. }
$$


6.5.4 THEOREM. If $f \in L^{p}, 1<p<\infty$, then

$$
\|\mathbf{S} f\|_{L^{p}} \leqslant A_{p}\|f\|_{L^{p}} .
$$

The constant $A_{p}$ depends only on $k$ and $p$.

Proof. By Theorem 6.5.1, if $f \in \Gamma_{\beta}$ for some $0<\beta<1$ and if $f$ has compact support then

$$
\mathbf{S} f=\operatorname{PVS} f+\frac{1}{2} f .
$$

In particular, (6.5.5) holds for compactly supported $f \in C^{\infty}$. The id?ntity operator is bounded on $L^{p}$ and, by Theorem (6.4.1), PVS is bounded on $L^{p}, 1<p<\infty$. Hence, by (6.5.5)

$$
\|\mathbf{S} f\|_{L^{p}} \leqslant A_{p}\|f\|_{L^{p}} \quad \text { for compactly supported } f \in C^{\infty} .
$$

Since the set of compactly supported $f \in C^{\infty}$ is dense in $L^{p}$, we obtain (6.5.6) for all $f \in L^{p}$.

\subsubsection{Theorem. Suppose $0<\beta<1,1<p<\infty$, and $f \in \Gamma_{\beta} \cap L^{p}$. Then}

$$
\|\mathbf{S} f\|_{\Gamma_{\beta}} \leqslant A_{p, \beta}^{\prime}\left(\|f\|_{\Gamma_{\beta}}+\|f\|_{L^{p}}\right),
$$

where $A_{p, \beta}^{\prime}$ depends only on $k, \beta$, and $p$.

Proof. By Theorem 6.5.1,

$$
\mathbf{S} f=\operatorname{PVS} f+\frac{1}{2} f .
$$

By Theorem 6.4.2, $\|\mathrm{PVS} f\|_{\Gamma_{\beta}} \leqslant A_{p, \beta}\left(\|f\|_{\Gamma_{\beta}}+\|f\|_{L^{p}}\right)$. Hence, by (6.5.8),

$$
\|\mathbf{S} f\|_{\Gamma_{\beta}} \leqslant\left(1+A_{p, \beta}\right)\left(\|f\|_{\Gamma_{\beta}}+\|f\|_{L^{p}}\right) .
$$

AcKNOWLEDGMENT. I wish to thank E. M. Stein for his guidance during the preparation of the doctoral dissertation on which this paper is based.

\section{REFERENCES}

[AhS] P. Ahern and R. Schneider, Holomorphic Lipschitz functions in pseudoconvex domains, Amer. J. Math. 101 (1979), 543-565.

[A1] W. Alt, Singuläre Integrale mit gemischten Homogenitäten auf Mannigfaltigkeiten und Andwendungen in der Funktionentheorie, Math. Z. 137 (1974), 227-256.

[BL] A. Bonami and N. Lohoué, Projecteurs de Bergman et Szegö pour une classe de domaines faiblement pseudo-convexes et estimations $L^{p}$, Compositio Math., 46 (1982), 159-226.

[CW] R. Coifman and G. Weiss, Analyse harmonique non-commutative sur certains espaces homogenes, Lecture Notes in Math., vol. 242, Springer-Verlag, 1971.

[Di] K. P. Diaz, The Szegö kernel as a singular integral kernel on a family of weakly pseudoconvex domains, Ph.D. dissertation, Princeton Univ., Princeton, N. J., 1986.

[FS] G. B. Folland and E. M. Stein, Estimates for the $\bar{\partial}_{b}$ complex and analysis on the Heisenberg group, Comm. Pure Appl. Math. 27 (1974), 429-522.

[Go] R. W. Goodman, Nilpotent Lie groups: structure and applications to analysis, Lecture Notes in Math., vol. 562 Springer-Verlag, 1976.

[GS] P. C. Greiner and E. M. Stein, On the solvability of some differential operators of type $\square_{b}$, Proc. Internat. Conf., (Cortona, Italy, 1976-1977), Scuola Norm. Sup. Pisa, Pisa, 1978, pp. 106-165.

[Ko] J. J. Kohn, Boundary behavior of $\bar{\partial}$ on weakly pseudo-convex manifolds of dimension two, J. Differential Geometry 6 (1972), 523-542.

[KoV] A. Korányi and S. Vági, Singular integrals on homogeneous spaces and some problems of classical analysis, Ann. Scuola Norm. Sup. Pisa 25 (1971), 575-648. 
[Kr] S. G. Krantz, Function theory of several complex variables, Wiley, 1982.

[NS] A. Nagel and E. M. Stein, Lectures on pseudo-differential operators: regularity theorems and applications to non-elliptic problems, Princeton Univ. Press, Princeton, N. J., 1979.

[NSW1] A. Nagel, E. M. Stein and S. Wainger, Boundary behavior of functions holomorphic in domains of finite type, Proc. Nat. Acad. Sci. U.S.A. 78 (1981), 6596-6599.

[NSW2] _. Balls and metrics defined by vector fields I: Basic properties, Acta Math. 155 (1985), 102-147.

[PS] D. H. Phong and E. M. Stein, Estimates for the Bergman and Szegö projections on strongly pseudo-convex domains, Duke Math. J. 44 (1977), 695-704.

[S1] E. M. Stein, Singular integrals and differentiability properties of functions, Princeton Univ. Press, Princeton, N. J., 1979.

[S2] __ Boundary behavior of holomorphic functions of several complex variables, Princeton Univ. Press, Princeton, N. J., 1972.

Department of Mathematics, Texas A \& M University, College Station, Texas 77843 